\title{
Paley-Wiener Isomorphism Over Infinite-Dimensional Unitary Groups
}

\author{
Oleh Lopushansky
}

\begin{abstract}
An analog of the Paley-Wiener isomorphism for the Hardy space with an invariant measure over infinite-dimensional unitary groups is described. This allows us to investigate on such space the shift and multiplicative groups, as well as, their generators and intertwining operators. We show applications to the Gauss-Weierstrass semigroups and to the Weyl-Schrödinger irreducible representations of complexified infinitedimensional Heisenberg groups.
\end{abstract}

Mathematics Subject Classification. 46T12, 46G20.

Keywords. Hardy spaces of infinitely many variables, Harmonic analysis on infinite-dimensional groups, Symmetric Fock spaces.

\section{Introduction}

The work deals with the Hardy space $H_{\chi}^{2}$ of square-integrable $\mathbb{C}$-valued functions with respect to a probability measure $\chi$ over the infinite-dimensional unitary group $U(\infty):=\bigcup\{U(m): m \in \mathbb{N}\}$, extended by unit $\mathbb{1}$, which irreducibly acts on a separable complex Hilbert space $E$ with an orthonormal basis $\left\{e_{m}\right\}$. Here, $U(m)$ is the subgroup of unitary $m \times m$-matrices endowed with Haar's measure $\chi_{m}$.

In what follows, $U(\infty)$ is densely embedded via a universal mapping $\pi$ into the space of virtual unitary matrices $\mathfrak{U}=\underset{\lim }{\longleftarrow} U(m)$ defined as the projective limit under Livšic's mappings $\pi_{m}^{m+1}: U(\overleftarrow{m+1}) \rightarrow U(m)$. The projective limit $\chi=\lim _{\longleftarrow} \chi_{m}$, such that each image-measure $\pi_{m}^{m+1}\left(\chi_{m+1}\right)$ is equal to $\chi_{m}$, is concentrated on the range $\pi(U(\infty))$ consisting of stabilized sequences (see $[18,20])$. The measure $\chi$ is invariant under right actions $[20$, n.4] . 
We refer to $[5,26]$ for applications of $\chi$ to stochastic processes. Needed properties of Hardy spaces $H_{\chi}^{2}$ can be found in [15]. Various cases of Hardy spaces in infinite-dimensional settings were considered in $[9,17]$.

Now, we briefly describe results. Using a unitarily weighted symmetric Fock space $\left(\Gamma_{\mathrm{w}},\langle\cdot \mid \cdot\rangle_{\mathrm{w}}\right)$ with a canonical orthogonal basis of symmetric tensor products $\left\{e_{\imath}^{\odot \lambda}\right\}$ of basis elements $\left\{e_{m}\right\} \subset E$ indexed by Young diagrams $\lambda$ and normalized by measure $\chi$, we find an orthogonal basis of polynomial $\left\{\phi_{l}^{\lambda}\right\}$ in $H_{\chi}^{2}$ such that the conjugate-linear mapping

$$
\Phi: \Gamma_{\mathrm{w}} \rightarrow H_{\chi}^{2}
$$

is a surjective isometry with one-to-one correspondence $e_{\imath}^{\odot \lambda} \rightleftarrows \phi_{\imath}^{\lambda}$. This allows us to establish in Theorem 2 an integral formula for a Fock-symmetric $\mathcal{F}$ transform

$$
\mathcal{F}: H_{\chi}^{2} \ni f \mapsto \widehat{f} \in H_{\mathrm{w}}^{2}
$$

where the Hilbert space $H_{\mathrm{w}}^{2}$, uniquely determined by $\Gamma_{\mathrm{w}}$, consists of HilbertSchmidt analytic entire functions on $E$. Thus, the $\mathcal{F}$-transform acts as an analog of the Paley-Wiener isomorphism over infinite-dimensional groups.

Furthermore, we investigate two different representations of the additive group $(E,+)$ over the Hardy space $H_{\chi}^{2}$ by shift and multiplicative groups. Theorem 3 states that the $\mathcal{F}$-transform is an intertwining operator between the multiplication group $M_{\mathrm{a}}^{\dagger}$ on $H_{\chi}^{2}$ and the shift group $T_{\mathrm{a}}$ on $H_{\mathrm{w}}^{2}$. On the other hand, Theorem 4 shows that $\mathcal{F}$ is the same between the shift group $T_{a}^{\dagger}$ on $H_{\chi}^{2}$ and the multiplication group $M_{\mathrm{a}^{*}}$ on $H_{\mathrm{w}}^{2}$. Integral formulas describing interrelations between their generators are established. In Theorem 5 suitable commutation relations are stated.

Applications to the Gauss-Weierstrass-type semigroups on $H_{\chi}^{2}$ are shown in Theorem 6. Another application to linear representations of complexified infinite-dimensional Heisenberg groups on $H_{\chi}^{2}$ in a Weyl-Schrödinger form is given in Theorem 7 .

Infinite-dimensional Heisenberg groups was considered in [16] by using reproducing kernel Hilbert spaces. The Schrödinger representation of infinitedimensional Heisenberg groups on $L_{\gamma}^{2}$ with respect to a Gaussian measure $\gamma$ over a real Hilbert space is described in [3] (see also earlier publications [1,2]).

In conclusion, we note that a motivation for this study was the following simple relations in the Hardy space $H_{\chi}^{2}$ over 1-dimensional group $U(1)=$ $\{u=\exp (\dot{\mathrm{i}} \vartheta): \vartheta \in[-\pi, \pi]\}$. In this case, $\left\{u^{n}: n \in \mathbb{Z}_{+}\right\}$is an orthonormal basis and $\Gamma_{\mathrm{w}} \simeq \ell^{2}$, since $\Phi^{*} f=\left(f_{n}\right) \in \ell^{2}$ for any $f \in H_{\chi}^{2}$ with Fourier coefficients $f_{n}=\int f(u) \bar{u}^{n} d u=\left\langle u^{n} \mid \Phi^{*} f\right\rangle_{\mathrm{w}}$. While, $\widehat{f}(x)=\int f(u) \exp (x \bar{u}) d u=$ $\sum f_{n} x^{n} / n !=\left\langle\varepsilon(x) \mid \Phi^{*} f\right\rangle_{\mathrm{w}}$, where $\varepsilon(x)=\left(x^{n} / n !\right) \in \ell^{2}$ for all $x \in \mathbb{C}$.

Moreover, the equalities $T_{a} \widehat{f}(x)=\int f(u) \exp [(x+a) \bar{u}] d u=\mathcal{F}\left(M_{a}^{\dagger} f\right)(x)$ with $M_{a}^{\dagger} f(u)=\exp (a \bar{u}) f(u)$ hold for all $x, a \in \mathbb{C}$. On the other hand, $\left(M_{\bar{a}} \widehat{f}\right)(x)$ $=\exp (x \bar{a}) \sum f_{n} x^{n} / n !=\sum\left(T_{\bar{a}}^{\dagger} f_{n}\right) x^{n} / n !$ with $T_{\bar{a}}^{\dagger} f_{n}=\sum_{k=0}^{n}\left(\begin{array}{l}n \\ k\end{array}\right) \bar{a}^{k} f_{n-k}$. Hence, 
$M_{\bar{a}} \widehat{f}=\mathcal{F}\left(T_{\bar{a}}^{\dagger} f\right)$, where $\left(T_{\bar{a}}^{\dagger} f\right)(u)=\sum\left(T_{\bar{a}}^{\dagger} f_{n}\right) u^{n}$. In result, $T_{a} M_{\bar{b}}=\exp (a \bar{b})$ $M_{\bar{b}} T_{a}$ for all $a, b \in \mathbb{C}$ and the Weyl-Schrödinger representation of Heisenberg's group from Theorem 7 retains a classic form.

The case $H_{\chi}^{2}$ over $m$-dimensional group $U(m)$ is similar with a proviso that the weighted Fock space $\Gamma_{\mathrm{w}}$ is normalized by $\left\|e_{\imath}^{\odot \lambda}\right\|_{\mathbb{w}}=\left(\begin{array}{c}n+m-1 \\ n\end{array}\right)^{-1 / 2}$ where $n=|\lambda|$ is a homogeneity degree of the basis polynomial $\phi_{\imath}^{\lambda}$ in $H_{\chi}^{2}$. Note that the normalization $\left\|e_{\imath}^{\odot \lambda}\right\|_{\mathrm{w}}=n !^{-1 / 2}$ with $n=|\lambda|$ leads to the case of Segal-Bargmann's space $H_{\gamma}^{2}$ with standard centered probability Gaussian measure $\gamma$ on $\mathbb{C}^{m}$.

\section{Hilbert-Schmidt Analyticity}

Let $E$ stand for a separable complex Hilbert space with scalar product $\langle\cdot \mid \cdot\rangle$ norm $\|\cdot\|$ and a fixed orthonormal basis $\left\{e_{k}: k \in \mathbb{N}\right\}$. Denote by $E_{\text {alg }}^{\otimes n}=$ $E \otimes{ }^{n \text { times }} \otimes E(n \in \mathbb{N})$ its algebraic tensor power consisted of the linear span of elements $\psi_{n}=x_{1} \otimes \cdots \otimes x_{n}$ with $x_{i} \in E(i=1, \ldots, n)$. Set $x^{\otimes n}:=$ $x \otimes{ }^{n \text { times }} \otimes x$. The symmetric algebraic tensor power $E_{\text {alg }}^{\odot n}=E \odot \cdots \odot E$ is defined to be the range of the projector $\mathfrak{s}_{n}: E_{\text {alg }}^{\otimes n} \ni \psi_{n} \mapsto x_{1} \odot \cdots \odot x_{n}$ with $x_{1} \odot \cdots \odot x_{n}:=(n !)^{-1} \sum_{\sigma} x_{\sigma(1)} \otimes \cdots \otimes x_{\sigma(n)}$ where $\sigma:\{1, \ldots, n\} \mapsto\{\sigma(1)$, $\ldots, \sigma(n)\}$ runs through all permutations. The symmetric algebraic Fock space is defined as the algebraic direct sum $\Gamma_{\text {alg }}=\sum_{n \in \mathbb{Z}_{+}} E_{\text {alg }}^{\odot n}$ with $E_{\text {alg }}^{\odot 0}=\mathbb{C}$.

Let $E_{\mathrm{h}}^{\otimes n}:=E \otimes_{\mathfrak{h}} \cdots \otimes_{\mathfrak{h}} E$ be the completion of $E_{\text {alg }}^{\otimes n}$ by Hilbertian norm $\left\|\psi_{n}\right\|_{\mathfrak{h}}=\left\langle\psi_{n} \mid \psi_{n}\right\rangle_{\mathfrak{h}}^{1 / 2}$ with $\left\langle\psi_{n} \mid \psi_{n}^{\prime}\right\rangle_{\mathfrak{h}}=\left\langle x_{1} \mid x_{1}^{\prime}\right\rangle \cdots\left\langle x_{n} \mid x_{n}^{\prime}\right\rangle$. Denote by $E_{\mathfrak{h}}^{\odot n}$ the range of continuous extension of $\mathfrak{s}_{n}$ on $E_{\mathrm{h}}^{\otimes n}$. As usual, the symmetric Fock space is defined to be the Hilbertian direct sum $\Gamma_{\mathfrak{h}}=\bigoplus_{n \in \mathbb{Z}_{+}} E_{\mathfrak{h}}^{\odot n}$.

Denote by $\lambda=\left(\lambda_{1}, \ldots, \lambda_{m}\right) \in \mathbb{Z}_{+}^{m}$ with $\lambda_{1} \geq \lambda_{2} \geq \cdots \geq \lambda_{m}$ a partition of $n \in \mathbb{N}$, that is, $n=|\lambda|$ where $|\lambda|:=\lambda_{1}+\cdots+\lambda_{m}$. Any $\lambda$ may be identified with Young's diagram of length $l(\lambda)=m$. Let $\mathbb{Y}$ denote all diagrams and $\mathbb{Y}_{n}=\{\lambda \in \mathbb{Y}:|\lambda|=n\}$. Assume that $\mathbb{Y}_{0}=\{\emptyset \in \mathbb{Y}:|\emptyset|=0\}$ and $l(\emptyset)=1$. Let $\mathbb{N}_{*}^{m}:=\left\{\imath=\left(\imath_{1}, \ldots, \imath_{m}\right) \in \mathbb{N}^{m}: \imath_{l} \neq \imath_{k}, \forall l \neq k\right\}$. For each $\lambda \in \mathbb{Y}$ we assign the constant

$$
C_{|\lambda|, l(\lambda)}:=\frac{(l(\lambda)-1) !|\lambda| !}{(l(\lambda)-1+|\lambda|) !} \leq 1 .
$$
tensors

The spaces $E_{\text {alg }}^{\odot n}$ and $\Gamma_{\text {alg }}$ may be generated by the basis of symmetric

$$
\begin{aligned}
e^{\odot \mathbb{Y}_{n}} & =\bigcup\left\{e_{\imath}^{\odot \lambda}:=e_{\imath_{1}}^{\otimes \lambda_{1}} \odot \cdots \odot e_{l_{l(\lambda)}}^{\otimes \lambda_{l(\lambda)}}:(\lambda, \imath) \in \mathbb{Y}_{n} \times \mathbb{N}_{*}^{l(\lambda)}\right\}, \\
e^{\odot \mathbb{Y}} & =\bigcup\left\{e^{\odot \mathbb{Y}_{n}}: n \in \mathbb{Z}_{+}\right\} \quad \text { with } \quad e_{\imath}^{\odot \emptyset}=1
\end{aligned}
$$


respectively. As is known [4, Sect. 2.2.2], norm of basis element in $\Gamma_{\mathfrak{h}}$ is equal to

$$
\left\|e_{\imath}^{\odot \lambda}\right\|_{\mathfrak{h}}^{2}=\frac{\lambda !}{|\lambda| !}, \quad \lambda !:=\lambda_{1} ! \cdots \lambda_{m} !
$$

Let us define a new Hilbertian norm on $\Gamma_{\text {alg }}$ by the equality $\|\cdot\|_{\mathrm{w}}=\langle\cdot \mid \cdot\rangle_{\mathrm{w}}^{1 / 2}$ where scalar product $\langle\cdot \mid \cdot\rangle_{\mathrm{w}}$ is determined via the orthogonal relations

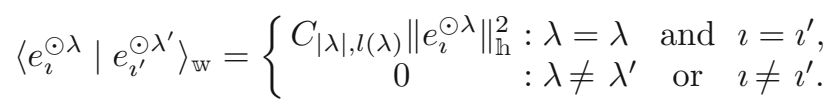

Denote by $E_{\mathrm{w}}^{\odot n}$ and $\Gamma_{\mathrm{w}}$ the appropriate completions of $E_{\text {alg }}^{\odot n}$ and $\Gamma_{\text {alg }}$, respectively. For any $\imath \in \mathbb{N}_{*}^{l(\lambda)}$ there corresponds in $E_{\mathrm{w}}^{\odot n}$ the $d$-dimension subspace with $d=C_{|\lambda|, l(\lambda)}^{-1}$, spanned by elements $\left\{e_{\imath}^{\odot \lambda}: \lambda \in \mathbb{Y}_{n}\right\}$. The Hilbertian orthogonal sum

$$
\Gamma_{\mathrm{w}}=\bigoplus_{n \in \mathbb{Z}_{+}} E_{\mathrm{w}}^{\odot n}
$$

endowed with $\langle\cdot \mid \cdot\rangle_{\mathrm{w}}$ we will call unitarily weighted symmetric Fock space.

Let $x=\sum e_{k} x_{k}$ be the Fourier series of $x \in E$ with coefficients $x_{k}=$ $\left\langle x \mid e_{k}\right\rangle$. We assign to any $(\lambda, \imath) \in \mathbb{Y}_{n} \times \mathbb{N}_{*}^{l(\lambda)}$ the $n$-homogenous HilbertSchmidt polynomial defined via the Fourier coefficients

$$
x_{\imath}^{\lambda}:=\left\langle x^{\otimes n} \mid e_{\imath}^{\odot \lambda}\right\rangle_{\mathrm{w}}=x_{\imath_{1}}^{\lambda_{1}} \ldots x_{\imath_{l(\lambda)}}^{\lambda_{l(\lambda)}}, \quad x \in E .
$$

Using the tensor multinomial theorem, we define in $\Gamma_{\mathrm{w}}$ the Fourier decomposition of exponential vectors (or coherent state vectors)

$$
\begin{aligned}
\varepsilon(x) & :=\bigoplus_{n \in \mathbb{Z}_{+}} \frac{x^{\otimes n}}{n !}=\bigoplus_{n \in \mathbb{Z}_{+}} \frac{1}{n !}\left(\sum_{k \in \mathbb{N}} e_{k} x_{k}\right)^{\otimes n} \\
& =\bigoplus_{n \in \mathbb{Z}_{+}} \frac{1}{n !} \sum_{(\lambda, \imath) \in \mathbb{Y}_{n} \times \mathbb{N}_{*}^{l(\lambda)}} \frac{n !}{\lambda !} e_{\imath}^{\odot \lambda} x_{\imath}^{\lambda}
\end{aligned}
$$

with respect to the basis $e^{\odot \mathbb{Y}}$. It is convergent in $\Gamma_{\mathrm{w}}$ in view of (1) and

$$
\begin{aligned}
\|\varepsilon(x)\|_{\mathbb{W}}^{2} & =\sum_{n \in \mathbb{Z}_{+}} \frac{1}{n !^{2}} \sum_{(\lambda, \imath) \in \mathbb{Y}_{n} \times \mathbb{N}_{*}^{l(\lambda)}}\left(\frac{n !}{\lambda !}\right)^{2}\left\|e_{\imath}^{\odot \lambda}\right\|_{\mathbb{W}}^{2}\left|x_{\imath}^{\lambda}\right|^{2} \\
& =\sum_{n \in \mathbb{Z}_{+}} \frac{1}{n !^{2}} \sum_{(\lambda, \imath)} \frac{n !}{\lambda !} C_{|\lambda|, l(\lambda)}\left|x_{\imath}^{\lambda}\right|^{2} \leq \sum_{n \in \mathbb{Z}_{+}} \frac{1}{n !} \sum_{(\lambda, \imath)} \frac{n !}{\lambda !}\left|x_{\imath}^{\lambda}\right|^{2} \\
& =\sum_{n \in \mathbb{Z}_{+}} \frac{1}{n !}\left(\sum_{k \in \mathbb{N}}\left|x_{k}\right|^{2}\right)^{n}=e^{\|x\|^{2}} .
\end{aligned}
$$

Particulary, (4) implies that the function $E \ni x \mapsto \varepsilon(x) \in \Gamma_{\mathrm{w}}$ is entire analytic. 
Definition 1. The space of $\mathbb{C}$-valued Hilbert-Schmidt entire analytic functions in variable $x \in E$, associating with the unitarily weighted symmetric Fock space $\Gamma_{\mathrm{w}}$, is defined to be

$$
H_{\mathrm{w}}^{2}:=\left\{\psi^{*}(x):=\langle\varepsilon(x) \mid \psi\rangle_{\mathrm{w}}: \psi \in \Gamma_{\mathrm{w}}\right\} \quad \text { with the norm }\left\|\psi^{*}\right\|=\|\psi\|_{\mathrm{w}} .
$$

Every function $\psi^{*}$ is entire analytic as the composition of $\varepsilon(\cdot)$ with $\langle\cdot \mid \psi\rangle_{\mathrm{w}}$. The subspace in $H_{\mathrm{w}}^{2}$ of $n$-homogenous Hilbert-Schmidt polynomials is defined to be

$$
H_{\mathrm{w}}^{2, n}=\left\{\psi_{n}^{*}(x)=\left\langle x^{\otimes n} \mid \psi_{n}\right\rangle_{\mathrm{w}}: \psi_{n} \in E_{\mathrm{w}}^{\odot n}\right\} .
$$

Evidently, $H_{\mathrm{w}}^{2}=\mathbb{C} \oplus H_{\mathrm{w}}^{2,1} \oplus H_{\mathrm{w}}^{2,2} \oplus \ldots$

It is important that $H_{\mathrm{w}}^{2}$ is uniquely determined by $\Gamma_{\mathrm{w}}$ since $\{\varepsilon(x): x \in E\}$ is total in $\Gamma_{\mathrm{w}}$. Similarly, for the subspace $H_{\mathrm{w}}^{2, n}$ which is uniquely determined by $E_{\mathrm{w}}^{\odot n}$, since $\left\{x^{\otimes n}: x \in E\right\}$ is total in $E_{\mathrm{w}}^{\odot n}$. The last totality follows from the polarization formula for symmetric tensor products

$$
e_{\imath}^{\odot \lambda}=\frac{1}{2^{n} n !} \sum_{\theta_{1}, \ldots, \theta_{n}= \pm 1} \theta_{1} \ldots \theta_{n} a^{\otimes n} \quad \text { with } \quad a=\sum_{i=1}^{l(\lambda)} \theta_{i} e_{\imath_{i}}^{\otimes \lambda_{i}}
$$

which is valid for all $e^{\odot \lambda} \in e^{\odot \mathbb{Y}_{n}}$ (see e.g. [11, Sect. 1.5]) Thus, the conjugatelinear isometries $\psi \mapsto \psi^{*}$ from $\Gamma_{\mathrm{w}}$ onto $H_{\mathrm{w}}^{2}$ and from $E_{\mathrm{w}}^{\odot n}$ onto $H_{\mathrm{w}}^{2, n}$ hold.

In conclusion, we can notice that every analytic function $\psi^{*} \in H_{\mathrm{w}}^{2}$ determined by $\psi=\bigoplus \psi_{n} \in \Gamma_{\mathrm{w}},\left(\psi_{n} \in E_{\mathrm{w}}^{\odot n}\right)$ has the Taylor expansion at zero

$$
\psi^{*}(x)=\sum_{n \in \mathbb{Z}_{+}} \frac{1}{n !} \sum_{(\lambda, \imath) \in \mathbb{Y}_{n} \times \mathbb{N}_{*}^{l(\lambda)}} \frac{\left\langle e_{\imath}^{\odot \lambda} \mid \psi_{n}\right\rangle_{\mathbb{w}}}{\left\|e_{\imath}^{\odot \lambda}\right\|_{\mathbb{w}}^{2}} x_{\imath}^{\lambda}, \quad x \in E
$$

that follows from (3). The function $\psi^{*}$ is entire Hilbert-Schmidt analytic [15, n.5].

Note that analytic functions of Hilbert-Schmidt types were also considered in $[10,14,21]$. More general classes of analytic functions associated with coherent sequences of polynomial ideals were described in [8].

\section{Hardy Space Over $U(\infty)$}

In what follows, we endow each group $U(m)$ with the probability Haar measure $\chi_{m}$ and assume that $U(m)$ is identified with its range with respect to the embedding $U(m) \ni u_{m} \mapsto\left[\begin{array}{cc}u_{m} & 0 \\ 0 & \mathbb{1}\end{array}\right] \in U(\infty)$. The Livšic transform from $U(m+1)$ onto $U(m)$ is described in [18, Proposition 0.1] and [20, Lemma 3.1] as the surjective Borel mapping

$$
\pi_{m}^{m+1}: u_{m+1}:=\left[\begin{array}{cc}
z_{m} & a \\
b & t
\end{array}\right] \longmapsto u_{m}:= \begin{cases}z_{m}-\left[a(1+t)^{-1} b\right] & : t \neq-1 \\
z_{m} & : t=-1\end{cases}
$$


The projective limit $\mathfrak{U}:=\lim U(m)$ under $\pi_{m}^{m+1}$ has surjective Borel projections $\pi_{m}: \mathfrak{U} \ni u \mapsto u_{m} \in U(m)$ such that $\pi_{m}=\pi_{m}^{m+1} \circ \pi_{m+1}$.

Consider a universal dense embedding $\pi: U(\infty) \uparrow \rightarrow \mathfrak{U}$ which to every $u_{m} \in U(m)$ assigns the stabilized sequence $u=\left(u_{k}\right)$ such that (see $[20, \mathrm{n} .4]$ )

$$
\pi: U(m) \ni u_{m} \mapsto\left(u_{k}\right) \in \mathfrak{U}, \quad u_{k}=\left\{\begin{array}{r}
\pi_{k}^{m}\left(u_{m}\right): k<m \\
u_{m}: k \geq m,
\end{array}\right.
$$

where $\pi_{k}^{m}:=\pi_{k}^{k+1} \circ \ldots \circ \pi_{m-1}^{m}$ for $k<m$ and $\pi_{k}^{m}$ is identity mapping for $k \geq m$. On its range $\pi(U(\infty))$, endowed with the Borel structure from $\mathfrak{U}$, we consider the inverse mapping

$$
\pi^{-1}: \mathfrak{U}_{\pi} \rightarrow U(\infty) \quad \text { where } \quad \mathfrak{U}_{\pi}:=\pi(U(\infty)) .
$$

The right action $\mathfrak{U}_{\pi} \ni u \mapsto u . g \in \mathfrak{U}_{\pi}$ with $g=(v, w) \in U(\infty) \times U(\infty)$ is defined by $\pi_{m}(u . g)=w^{-1} \pi_{m}(u) v$ where $m$ is so large that $g=(v, w) \in$ $U(m) \times U(m)$.

Following [18, n.3.1], [20, Lemma 4.8] via the Kolmogorov consistency theorem (see e.g. [19, Theorem 1], [24, Corollary 4.2]) we uniquely define on $\mathfrak{U}=\lim U(m)$ the probability measure $\chi:=\lim _{\text {} \chi_{m}}$ such that each image-

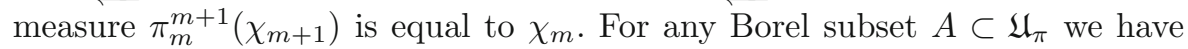
$\pi_{m+1}(A) \subseteq\left(\pi_{m}^{m+1}\right)^{-1}\left[\pi_{m}(A)\right]$, because $\pi_{m}=\pi_{m}^{m+1} \circ \pi_{m+1}$. It follows that $\left(\chi_{m} \circ \pi_{m}\right)(A)=\pi_{m}^{m+1}\left(\chi_{m+1}\right)\left[\pi_{m}(A)\right]=\chi_{m+1}\left[\left(\pi_{m}^{m+1}\right)^{-1}\left[\pi_{m}(A)\right]\right] \geq\left(\chi_{m+1} \circ\right.$ $\left.\pi_{m+1}\right)(A)$. Hence, $\chi$ satisfies the condition

$$
\chi(A)=\inf \left(\chi_{m} \circ \pi_{m}\right)(A)=\lim \chi_{m}(A)
$$

and therefore the projective $\operatorname{limit} \lim \chi_{m}$ exists on $\mathfrak{U}_{\pi}$ via the well known Prohorov theorem [6, Theorem IX.52]. Moreover, it is a Radon probability measure concentrated on $\mathfrak{U}_{\pi}[24$, Theorem 4.1]. By the known portmanteau theorem [13, Theorem 13.16] and Fubini's theorem the invariance of Haar measures $\chi_{m}$ together with (7) yield the following invariance properties under the right action

$$
\begin{aligned}
\int f(u . g) d \chi(u) & =\int f(u) d \chi(u), \quad g \in U(\infty) \times U(\infty), \quad f \in L_{\gamma}^{\infty}, \\
\int f d \chi & =\int d \chi(\mathfrak{u}) \int_{U(m) \times U(m)} f(\mathfrak{u} . g) d\left(\chi_{m} \otimes \chi_{m}\right)(g),
\end{aligned}
$$

where $L_{\chi}^{\infty}$ stands for the space of all $\chi$-essentially bounded complex-valued

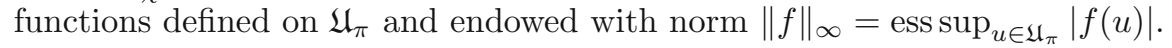

Let $L_{\chi}^{2}$ be the space of square-integrable $\mathbb{C}$-valued functions $f$ on $\mathfrak{U}_{\pi}$ with norm

$$
\|f\|_{\chi}=\langle f \mid f\rangle_{\chi}^{1 / 2} \quad \text { where }\langle f \mid f\rangle_{\chi}:=\int f_{1} \bar{f}_{2} d \chi
$$

The embedding $L_{\chi}^{\infty} \hookrightarrow L_{\chi}^{2}$ holds, moreover, $\|f\|_{\chi} \leq\|f\|_{\infty}$ for all $f \in L_{\chi}^{\infty}$. 
To given the $E$-valued mapping $\mathfrak{U}_{\pi} \ni u \mapsto \pi^{-1}(u) e_{1}$, we can well-define the Borel $\chi$-essentially bounded functions in the variable $u \in \mathfrak{U}_{\pi}$,

$$
\phi_{k}:=\phi_{e_{k}}, \quad \phi_{e_{k}}(u)=\left\langle\pi^{-1}(u) e_{1} \mid e_{k}\right\rangle, \quad k \in \mathbb{N},
$$

which do not depend on the choice of $e_{1}$ in $\bigcup S(m)$ where $S(m)$ is the $m$-dimensional unit sphere in $E$ [15, n.3]. The uniqueness of $\phi_{x}(u)=\left\langle\pi^{-1}(u) e_{1} \mid x\right\rangle$ with $x \in E$ results from the total embedding $\pi: U(\infty) \rightarrow \mathfrak{U}$. From (6) it follows that $\pi^{-1} \circ \pi_{m}^{-1}$ coincides with the embedding $U(m) \leftrightarrow U(\infty)$. Hence, by $(7)$ and the portmanteau theorem there exist the limit

$$
\int \phi_{x} d \chi=\lim _{m \rightarrow \infty} \int_{U(m)} \phi_{x} d\left(\chi_{m} \circ \pi_{m}\right)=\lim _{m \rightarrow \infty} \int_{U(m)}\left(\phi_{x} \circ \pi_{m}^{-1}\right) d \chi_{m}
$$

i.e., $\phi_{x} \in L_{\chi}^{\infty}$ for any $\phi_{x}(u)=\left\langle\pi^{-1}(u) e_{1} \mid x\right\rangle$ with $x \in E$.

By formula (5) to every $e_{\imath}^{\odot \lambda} \in e^{\odot \mathbb{Y}_{n}}$ there uniquely corresponds the Borel function from $L_{\chi}^{\infty}$

$$
\phi_{\imath}^{\lambda}(u):=\left\langle\left[\pi^{-1}(u) e_{1}\right]^{\otimes n} \mid e_{\imath}^{\odot \lambda}\right\rangle_{\mathbb{w}}=\phi_{\imath_{1}}^{\lambda_{1}}(u) \ldots \phi_{l_{l(\lambda)}}^{\lambda_{l(\lambda)}}(u)
$$

in the variable $u \in \mathfrak{U}_{\pi}$. It follows that the orthogonal basis $e^{\odot \mathbb{Y}}$ of elements $e_{\imath}^{\odot \lambda}=e_{\imath_{1}}^{\otimes \lambda_{1}} \odot \cdots \odot e_{\imath_{m}}^{\otimes \lambda_{m}}$, indexed by $\lambda=\left(\lambda_{1}, \ldots, \lambda_{m}\right) \in \mathbb{Y}$ and $\imath=\left(\imath_{1}, \ldots, \imath_{m}\right)$ $\in \mathbb{N}_{*}^{m}$ with $m=l(\lambda)$, uniquely determines the systems of Borel $\chi$-essentially bounded functions in the variable $u \in \mathfrak{U}_{\pi}$,

$$
\begin{aligned}
\phi^{\mathbb{Y}_{n}} & =\bigcup\left\{\phi_{\imath}^{\lambda}:=\phi_{\imath_{1}}^{\lambda_{1}} \cdots \phi_{\imath_{m}}^{\lambda_{m}}:(\lambda, \imath) \in \mathbb{Y}_{n} \times \mathbb{N}_{*}^{m}, m=l(\lambda)\right\}, \\
\phi^{\mathbb{Y}} & =\bigcup\left\{\phi^{\mathbb{Y}_{n}}: n \in \mathbb{Z}_{+}\right\} \quad \text { with } \quad \phi_{\imath}^{\emptyset} \equiv 1 .
\end{aligned}
$$

Definition 2. The Hardy space $H_{\chi}^{2}$ is defined as the closed complex linear span of $\phi^{\mathbb{Y}}$ endowed with $L_{\chi}^{2}$-norm.

The following assertion is proved in [15, Theorem 3.2].

Theorem 1. The system of Borel functions $\phi^{\mathbb{Y}}$ forms an orthogonal basis in $H_{\chi}^{2}$ such that

$$
\left\|\phi_{\imath}^{\lambda}\right\|_{\chi}=C_{|\lambda|, l(\lambda)}^{1 / 2}\left\|e_{\imath}^{\odot \lambda}\right\|_{\mathfrak{h}}, \quad \lambda \in \mathbb{Y}, \quad \imath \in \mathbb{N}_{*}^{l(\lambda)} .
$$

Define the subspace $H_{\chi}^{2, n} \subset H_{\chi}^{2}$ for any $n \in \mathbb{N}$ to be the closed linear span of the subsystem $\phi^{\mathbb{Y}_{n}}$. Theorem 1 implies that $H_{\chi}^{2, n} \perp H_{\chi}^{2, m}$ in $L_{\chi}^{2}$ for any $n \neq m$. This provides the orthogonal decomposition

$$
H_{\chi}^{2}=\mathbb{C} \oplus H_{\chi}^{2,1} \oplus H_{\chi}^{2,2} \oplus \cdots .
$$




\section{Fock-Symmetric $\mathcal{F}$-Transform}

The one-to-one correspondence $e_{\imath}^{\odot \lambda} \rightleftarrows \phi_{\imath}^{\lambda}$ allows us to define via the change of orthonormal bases

$$
\Phi: \Gamma_{\mathrm{w}} \ni e_{\imath}^{\odot \lambda}\left\|e_{\imath}^{\odot \lambda}\right\|_{\mathbb{w}}^{-1} \mapsto \phi_{\imath}^{\lambda}\left\|\phi_{\imath}^{\lambda}\right\|_{\chi}^{-1} \in H_{\chi}^{2}, \quad \lambda \in \mathbb{Y}, \quad \imath \in \mathbb{N}_{*}^{l(\lambda)}
$$

the isometric conjugate-linear mapping $\Phi: \Gamma_{\mathrm{w}} \rightarrow H_{\chi}^{2}$. The adjoint mapping $\Phi^{*}: H_{\chi}^{2} \rightarrow \Gamma_{\mathrm{w}}$ is defined by $\left\langle\Phi e_{\imath}^{\odot \lambda} \mid f\right\rangle_{\chi}=\left\langle e_{\imath}^{\odot \lambda} \mid \Phi^{*} f\right\rangle_{\mathrm{w}}$ with $f \in H_{\chi}^{2}$. The suitable Fourier decomposition has the form

$$
\Phi \psi=\sum_{(\lambda, \imath) \in \mathbb{Y} \times \mathbb{N}_{*}^{l(\lambda)}} \hat{\psi}_{(\lambda, \imath)} \phi_{\imath}^{\lambda}\left\|\phi_{\imath}^{\lambda}\right\|_{\chi}^{-1}, \quad \hat{\psi}_{(\lambda, \imath)}:=\left\langle e_{\imath}^{\odot \lambda} \mid \psi\right\rangle_{\mathbb{w}}\left\|e_{\imath}^{\odot \lambda}\right\|_{\mathbb{W}}^{-1}
$$

for any $\psi \in \Gamma_{\mathrm{w}}$. In particular, the equality $\Phi x=\sum x_{k} \phi_{k}$ is valid for all $x \in E$. This gives the equalities

$$
\|\Phi x\|_{\chi}^{2}=\sum\left|x_{k}\right|^{2}=\|x\|^{2}, \quad x \in E .
$$

Using this, we will examine the composition of $\Phi$ with the $\Gamma_{\mathrm{w}}$-valued function $\varepsilon: E \ni x \mapsto \varepsilon(x)$. Its correctness justifies the following assertion that substantially uses the $L_{\chi}^{\infty}$-valued function

$$
\phi_{x}: \mathfrak{U}_{\pi} \ni u \mapsto(\Phi x)(u)=\sum x_{k} \phi_{k}(u)
$$

which is linear in the variable $x \in E$.

Similarly to the known case of Wiener spaces, the function $\Phi x$ can be seen as a group analog of the Paley-Wiener map (see e.g. [12, n.4.4] or [23]).

Lemma 1. The composition $\Phi \varepsilon(x)$, which is understood as the function

$$
[\Phi \varepsilon(x)](u): \mathfrak{U}_{\pi} \ni u \mapsto \exp \left(\phi_{x}(u)\right),
$$

takes values in $L_{\chi}^{\infty}$ for all $x \in E$.

Proof. Applying $\Phi$ to the Fourier decomposition (3), we obtain

$$
\Phi \varepsilon(x)=\sum_{n \in \mathbb{Z}_{+}} \frac{1}{n !} \sum_{(\lambda, \imath) \in \mathbb{Y}_{n} \times \mathbb{N}_{*}^{l(\lambda)}} \frac{n !}{\lambda !} x_{\imath}^{\lambda} \phi_{\imath}^{\lambda}=\sum_{n \in \mathbb{Z}_{+}} \frac{1}{n !}\left(\sum_{k \in \mathbb{N}} x_{k} \phi_{k}\right)^{n}=\exp \left(\phi_{x}\right) .
$$

It directly follows that $\|\Phi \varepsilon(x)\|_{\infty} \leq \exp \left\|\phi_{x}\right\|_{\infty}$.

Theorem 2. For every $f=\sum f_{n} \in H_{\chi}^{2},\left(f_{n} \in H_{\chi}^{2, n}\right)$ the entire analytic function $\widehat{f}(x):=\left\langle\varepsilon(x) \mid \Phi^{*} f\right\rangle_{\mathrm{w}}$ in the variable $x \in E$ and its Taylor coefficients at origin have the integral representations

$$
\widehat{f}(x)=\int \exp \left(\bar{\phi}_{x}\right) f d \chi \quad \text { and } \quad d_{0}^{n} \widehat{f}(x)=\int \bar{\phi}_{x}^{n} f_{n} d \chi,
$$

respectively. The mapping $\mathcal{F}: H_{\chi}^{2} \ni f \mapsto \widehat{f} \in H_{\mathrm{w}}^{2}$ (regarded as a Fock-symmetric $\mathcal{F}$-transform) provides the isometries

$$
H_{\chi}^{2} \simeq H_{\mathrm{w}}^{2} \quad \text { and } \quad H_{\chi}^{2, n} \simeq H_{\mathrm{w}}^{2, n} .
$$


Proof. First recall that the $\Gamma_{\mathrm{W}}$-valued function $\varepsilon(\cdot)$ is entire analytic on $E$, therefore $\widehat{f}$ is the same, as the composition of $\varepsilon(\cdot)$ with $\left\langle\cdot \mid \Phi^{*} f\right\rangle_{\mathrm{w}}$. Farther on, consider the Fourier decomposition with respect to the basis $\phi^{\mathbb{Y}}$,

$$
f=\sum_{n \in \mathbb{Z}_{+}} f_{n}=\sum_{\substack{n \in \mathbb{Z}_{+} \\(\lambda, \imath) \in \mathbb{Y}_{n} \times \mathbb{N}_{*}^{l(\lambda)}}} \frac{\hat{f}_{\lambda, \imath, n} \bar{\phi}_{l}^{\lambda}}{\left\|\phi_{\imath}^{\lambda}\right\|_{\chi}}, \quad \hat{f}_{\lambda, \imath, n}=\frac{1}{\left\|\phi_{\imath}^{\lambda}\right\|_{\chi}} \int f \bar{\phi}_{\imath}^{\lambda} d \chi .
$$

Applying $\Phi^{*}$ to $f$ in this decomposition and substituting $\hat{f}_{\lambda, \imath, n}$ into $\widehat{f}$, we obtain

$$
\begin{aligned}
\widehat{f}(x) & =\sum_{n \in \mathbb{Z}_{+}} \frac{1}{n !} \sum_{(\lambda, \imath) \in \mathbb{Y}_{n} \times \mathbb{N}_{*}^{l(\lambda)}} \frac{n !}{\lambda !} \frac{\hat{f}_{\lambda, \imath, n}\left\langle e_{\imath}^{\odot \lambda} \mid e_{\imath}^{\odot \lambda}\right\rangle_{\mathbb{w}} x_{\imath}^{\lambda}}{\left\|e_{\imath}^{\odot \lambda}\right\|_{\mathbb{w}}} \\
& =\int \sum_{n \in \mathbb{Z}_{+}} \frac{1}{n !}\left(\sum_{(\lambda, \imath) \in \mathbb{Y}_{n} \times \mathbb{N}_{*}^{l(\lambda)}} \frac{n !}{\lambda !} x_{\imath}^{\lambda} \bar{\phi}_{\imath}^{\lambda}\right) f d \chi=\int \exp \left(\bar{\phi}_{x}\right) f d \chi
\end{aligned}
$$

where the last equality is valid by Lemma 1 . It particularly follows that for $y=\alpha x$,

$$
\widehat{f}(y)=\int \exp \left(\bar{\phi}_{\alpha x}\right) f d \chi=\sum \alpha^{n} \int \frac{\bar{\phi}_{x}^{n}}{n !} f_{n} d \chi, \quad \alpha \in \mathbb{C} .
$$

Differentiating $\widehat{f}$ at $y=0$ and using the $n$-homogeneity of derivatives, we obtain

$$
d_{0}^{n} \widehat{f}(x)=\left.\frac{d^{n}}{d \alpha^{n}} \sum \alpha^{n} \int \frac{\bar{\phi}_{x}^{n}}{n !} f_{n} d \chi\right|_{\alpha=0}=\int \bar{\phi}_{x}^{n} f_{n} d \chi .
$$

Finally, we notice that the isometry $H_{\chi}^{2} \simeq H_{\mathrm{w}}^{2}$ holds, since the isometry $\Phi^{*}$ is surjective. In the case of polynomials we similarly get $H_{\chi}^{2, n} \simeq H_{\mathrm{w}}^{2, n}$.

Note that a different integral formula for analytic functions employing Wiener measures on infinite-dimensional Banach spaces was presented in [22].

\section{Exponential Creation and Annihilation Groups}

Let us define the linear mapping $\mathfrak{j}_{n}: E_{\mathrm{w}}^{\odot n} \rightarrow E_{\mathbb{h}}^{\odot n}$ to be the continuous extension of identity mapping acting on the dense subspace $E_{\mathrm{alg}}^{\odot n} \subset E_{\mathrm{w}}^{\odot n} \cap E_{\mathrm{h}}^{\odot n}$. Such continuous extension $\mathfrak{j}_{n}$ is a contractive injection with dense range. In fact, it suffices to expand elements from $E_{\mathbb{w}}^{\odot n}$ and $E_{\mathbb{h}}^{\odot n}$ into the Fourier series with respect to orthogonal basis $e^{\odot \mathbb{Y}_{n}}$ and apply the inequality

$$
\left\|e_{\imath}^{\odot \lambda}\right\|_{\mathbb{W}}^{2}=C_{|\lambda|, l(\lambda)}\left\|e_{\imath}^{\odot \lambda}\right\|_{\mathfrak{h}}^{2} \leq\left\|e_{\imath}^{\odot \lambda}\right\|_{\mathbb{h}}^{2}, \quad \lambda \in \mathbb{Y}_{n}
$$

which follows from Theorem 1, taking into account the inequality (1). Using subsequently that $E_{\mathrm{h}}^{\odot n}$ is reflexive, we obtain that its adjoint operator 
$\mathrm{j}_{n}^{*}: E_{\mathrm{h}}^{\odot n} \rightarrow E_{\mathrm{w}}^{\odot n}$ is a contractive injection with dense range. Thus, the mapping $\mathfrak{j}_{n}$ is also injective. Moreover, $E_{\mathrm{h}}^{\odot n} \stackrel{\mathfrak{j}_{n}^{*}}{\rightarrow} E_{\mathrm{w}}^{\odot n} \stackrel{\mathfrak{j}_{n}}{\rightarrow} E_{\mathrm{h}}^{\odot n}$ forms a Gelfand triple. Particularly, the operator $\mathfrak{s}_{n}$ possesses continuous extension on $E_{\mathrm{w}}^{\odot n}$.

Using this, we consider the linear operator

$$
\mathfrak{s}_{n / m}:=\mathfrak{s}_{n} \circ\left(\mathfrak{j}_{m} \otimes \mathfrak{j}_{n-m}\right) \quad \text { with } \quad m \leq n
$$

defined to be $\phi_{m} \odot \psi_{n-m}=\mathfrak{s}_{n / m}\left(\phi_{m} \otimes \psi_{n-m}\right) \in E_{\mathrm{w}}^{\odot n}$ for all $\phi_{m} \in E_{\mathrm{w}}^{\odot m}$, $\psi_{n-m} \in E_{\mathbb{w}}^{\odot(n-m)}$.

Lemma 2. The mapping $\mathfrak{s}_{n / m}$ from $E_{\mathrm{w}}^{\odot m} \otimes_{\mathrm{h}} E_{\mathbb{w}}^{\odot(n-m)}$ to $E_{\mathrm{w}}^{\odot n}$ is a contractive injection with dense range.

Proof. Expand elements of $E_{\mathrm{w}}^{\odot m} \otimes_{\mathbb{h}} E_{\mathrm{W}}^{\odot(n-m)}$ with respect to $e_{\imath}^{\odot \lambda} \otimes e_{\jmath}^{\odot \mu}$ for all $\lambda, \mu \in \mathbb{Y}, \imath \in \mathbb{N}^{l(\lambda)}, \jmath \in \mathbb{N}^{l(\mu)}$ such that $|\lambda|=m,|\mu|=n-m$. Using (9), we have

$$
\begin{aligned}
\left\|e_{\imath}^{\odot \lambda} \otimes e_{\jmath}^{\odot \mu}\right\|_{E_{\mathrm{w}}^{\odot m} \otimes_{\mathrm{h}} E_{\mathrm{w}}^{\odot(n-m)}} & =\left\|e_{\imath}^{\odot \lambda}\right\|_{\mathrm{w}}\left\|e_{\jmath}^{\odot \mu}\right\|_{\mathrm{w}} \\
& \leq\left\|e_{\imath}^{\odot \lambda}\right\|_{\mathrm{h}}\left\|e_{\jmath}^{\odot \mu}\right\|_{\mathrm{h}}=\left\|e_{\imath}^{\odot \lambda} \otimes e_{\jmath}^{\odot \mu}\right\|_{\mathrm{h}} .
\end{aligned}
$$

As above, it implies that the mapping $\mathfrak{j}_{m} \otimes \mathfrak{j}_{n-m}: E_{\mathbb{w}}^{\odot m} \otimes_{\mathbb{h}} E_{\mathbb{W}}^{\odot(n-m)} \rightarrow E_{\mathfrak{h}}^{\otimes n}$, defined to be the continuous extension of identity mapping on $E_{\mathrm{alg}}^{\odot m} \otimes E_{\mathrm{alg}}^{\odot(n-m)}$, is a contractive injection. Using subsequently that $E_{\mathfrak{h}}^{\odot m} \otimes_{\mathfrak{h}} E_{\mathfrak{h}}^{\odot(n-m)}$ is reflexive, we get the Gelfand triple

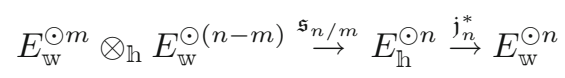

where injections are contractive and have dense ranges.

Lemma 3. The $\Gamma_{\mathrm{w}}$-valued function, defined on $\{\varepsilon(x): x \in E\}$ by

$$
\mathcal{T}_{\mathrm{a}} \varepsilon(x)=\varepsilon(x+\mathrm{a}),
$$

has a unique linear extension $\mathcal{T}_{\mathrm{a}}: \Gamma_{\mathrm{w}} \ni \psi \mapsto \mathcal{T}_{\mathrm{a}} \psi \in \Gamma_{\mathrm{w}}$ such that

$$
\left\|\mathcal{T}_{\mathrm{a}} \psi\right\|_{\mathrm{w}}^{2} \leq \exp \left(\|\mathrm{a}\|^{2}\right)\|\psi\|_{\mathrm{w}}^{2} \quad \text { and } \quad \mathcal{T}_{\mathrm{a}+\mathrm{b}}=\mathcal{T}_{\mathrm{a}} \mathcal{T}_{\mathrm{b}}=\mathcal{T}_{\mathrm{b}} \mathcal{T}_{\mathrm{a}} \quad \text { for all } \mathrm{a}, \mathrm{b} \in E .
$$

Proof. Let us define the creation operators $\delta_{\mathrm{a}, n}^{m}: E_{\mathrm{w}}^{\odot(n-m)} \rightarrow E_{\mathrm{w}}^{\odot n}(m \leq n)$ as

$$
\delta_{\mathrm{a}, n}^{m} x^{\otimes(n-m)}:=\mathfrak{s}_{n / m}\left[\mathrm{a}^{\otimes m} \otimes x^{\otimes(n-m)}\right]=\left.\frac{(n-m) !}{n !} \frac{d^{m}(x+t \mathrm{a})^{\otimes n}}{d t^{m}}\right|_{t=0}
$$

for all a, $x \in E$. Note that the second equality in (10) follows from the binomial formula for symmetric tensor elements $(x+t a)^{\otimes n}=\sum_{m=0}^{n}\left(\begin{array}{l}n \\ m\end{array}\right)(t a)^{\otimes m} \odot$ $x^{\otimes(n-m)}$. Put $\delta_{\mathrm{a}, n}^{0}=1$. If a $=0$ then $\delta_{0, n}^{m}=0$. Summing over $n \geq m$ with coefficients $1 /(n-m)$ !, we get

$$
\delta_{\mathrm{a}}^{m} \varepsilon(x)=\left.\frac{d^{m} \varepsilon(x+t \mathrm{a})}{d t^{m}}\right|_{t=0}=\bigoplus_{n \geq m} \frac{\mathfrak{s}_{n / m}\left[\mathrm{a}^{\otimes m} \otimes x^{\otimes(n-m)}\right]}{(n-m) !}, \quad t \in \mathbb{C} .
$$


This series is convergent, since by Lemma 2 and (4) the inequality

$$
\left\|\delta_{\mathrm{a}}^{m} \varepsilon(x)\right\|_{\mathbb{w}} \leq\|\mathrm{a}\|^{m}\left\|\bigoplus_{n \geq m} \frac{x^{\otimes(n-m)}}{(n-m) !}\right\|_{\mathbb{w}}=\|\mathrm{a}\|^{m}\|\varepsilon(x)\|_{\mathbb{w}}
$$

holds. From (11) and the tensor binomial formula mentioned above it follows that

$$
\bigoplus_{m=0}^{n} \frac{1}{m !} \delta_{\mathrm{a}, n}^{m} \frac{x^{\otimes(n-m)}}{(n-m) !}=\bigoplus_{m=0}^{n} \frac{\mathrm{a}^{\otimes m} \odot x^{\otimes(n-m)}}{m !(n-m) !}=\frac{(x+\mathrm{a})^{\otimes n}}{n !} .
$$

Summing over $n \in \mathbb{Z}_{+}$with coefficients $1 / n$ ! and using (11), we obtain

$$
\begin{aligned}
\mathcal{T}_{\mathrm{a}} \varepsilon(x) & =\bigoplus_{n \in \mathbb{Z}_{+}} \sum_{m=0}^{n} \frac{1}{m !} \delta_{\mathrm{a}, n}^{m} \frac{x^{\otimes(n-m)}}{(n-m) !} \\
& =\sum_{m \in \mathbb{Z}_{+}} \frac{1}{m !} \bigoplus_{n \geq m} \delta_{\mathrm{a}, n}^{m} \frac{x^{\otimes(n-m)}}{(n-m) !}=\exp \left(\delta_{\mathrm{a}}\right) \varepsilon(x) .
\end{aligned}
$$

The inequalities (4) and (12) yield $\left\|\mathcal{T}_{\mathrm{a}} \varepsilon(x)\right\|_{\mathbb{w}}^{2} \leq \exp \left(\|\mathrm{a}\|^{2}\right)\|\varepsilon(x)\|_{\mathbb{w}}^{2}$. Taking into account the totality of $\{\varepsilon(x): x \in E\}$, this inequality implies the required inequality on $\Gamma_{\mathrm{w}}$. It also follows that $\mathcal{T}_{\mathrm{a}+\mathrm{b}}=\mathcal{T}_{\mathrm{a}} \mathcal{T}_{\mathrm{b}}=\mathcal{T}_{\mathrm{b}} \mathcal{T}_{\mathrm{a}}$, since $\delta_{\mathrm{a}+\mathrm{b}}=\delta_{\mathrm{a}}+\delta_{\mathrm{b}}$ for all $\mathrm{a}, \mathrm{b} \in E$ by linearity of creation operators. This ends the proof.

We define the adjoint operators $\delta_{\mathrm{a}, n}^{* m}: E_{\mathrm{w}}^{\odot n} \ni \psi_{n} \rightarrow \delta_{\mathrm{a}, n}^{* m} \psi_{n} \in E_{\mathrm{w}}^{\odot(n-m)}$ as

$$
\left\langle\delta_{\mathrm{a}, n}^{m} x^{\otimes(n-m)} \mid \psi_{n}\right\rangle_{\mathbb{w}}=\left\langle x^{\otimes(n-m)} \mid \delta_{\mathrm{a}, n}^{* m} \psi_{n}\right\rangle_{\mathbb{w}}, \quad \mathrm{a}, x \in E
$$

for $n \geq m$. It immediately follows that for every $\psi_{n-m} \in E_{\mathrm{w}}^{\odot(n-m)}$ and $x \in E$,

$$
\begin{aligned}
\left\langle\delta_{\mathrm{a}, n}^{* m} x^{\otimes n} \mid \psi_{n-m}\right\rangle_{\mathrm{w}} & =\left\langle x^{\otimes n} \mid \delta_{\mathrm{a}, n}^{m} \psi_{n-m}\right\rangle_{\mathrm{w}}=\left\langle x^{\otimes n} \mid \mathrm{a}^{\otimes m} \odot \psi_{n-m}\right\rangle_{\mathrm{w}} \\
& =\langle x \mid \mathrm{a}\rangle^{m}\left\langle x^{\otimes(n-m)} \mid \psi_{n-m}\right\rangle_{\mathbb{w}} .
\end{aligned}
$$

Using $\delta_{\mathrm{a}, n}^{* m}$, we can uniquely define a $\Gamma_{\mathrm{w}}$-valued function $\mathcal{T}_{\mathrm{a}}^{*}$ by the equalities

$$
\mathcal{T}_{\mathrm{a}}^{*} \varepsilon(x)=\exp \left(\delta_{\mathrm{a}}^{*}\right) \varepsilon(x)=\sum_{m \in \mathbb{Z}_{+}} \frac{\delta_{\mathrm{a}}^{* m} \varepsilon(x)}{m !}, \quad \delta_{\mathrm{a}}^{* m} \varepsilon(x):=\bigoplus_{n \geq m} \frac{\delta_{\mathrm{a}, n}^{* m} x^{\otimes n}}{n !}
$$

for all a, $x \in E$. Taking into account Lemma 3, we obtain the following claim.

Lemma 4. The $\Gamma_{\mathrm{w}}$-valued function $\mathcal{T}_{\mathrm{a}}{ }^{*}$, defined by (14), possesses a unique linear extension $\mathcal{T}_{\mathrm{a}}^{*}: \Gamma_{\mathrm{w}} \ni \psi \mapsto \mathcal{T}_{\mathrm{a}}^{*} \psi \in \Gamma_{\mathrm{w}}$ such that

$\left\|\mathcal{T}_{\mathrm{a}}^{*} \psi\right\|_{\mathrm{w}}^{2} \leq \exp \left(\|\mathrm{a}\|^{2}\right)\|\psi\|_{\mathrm{w}}^{2} \quad$ and $\quad \mathcal{T}_{\mathrm{a}+\mathrm{b}}^{*}=\mathcal{T}_{\mathrm{a}}^{*} \mathcal{T}_{\mathrm{b}}^{*}=\mathcal{T}_{\mathrm{b}}{ }^{*} \mathcal{T}_{\mathrm{a}}^{*} \quad$ for all $\quad \mathrm{a}, \mathrm{b} \in E$.

Definition 3. We will call the $\Gamma_{\mathrm{w}}$-valued functions $\mathcal{T}_{\mathrm{a}}$ and $\mathcal{T}_{\mathrm{a}}^{*}$ in variable a $\in E$ the exponential creation and annihilation groups, respectively. 


\section{Intertwining Properties of $\mathcal{F}$-Transform}

Let us define on the space $H_{\chi}^{2}$ the multiplicative group $M_{\mathrm{a}}^{\dagger}: E \ni$ a $\mapsto M_{\mathrm{a}}^{\dagger}$ to be

$$
M_{\mathrm{a}}^{\dagger} f(u)=\exp \left[\bar{\phi}_{\mathrm{a}}(u)\right] f(u), \quad f \in H_{\chi}^{2}, \quad u \in \mathfrak{U}_{\pi} .
$$

It can be considered as a linear representation of the additive group $(E,+)$. By Lemma 1 the function $u \mapsto \exp \left[\bar{\phi}_{\mathrm{a}}(u)\right]$ with a fixed a belongs to $L_{\chi}^{\infty}$. Hence, $M_{\mathrm{a}}^{\dagger}$ is continuous on $H_{\chi}^{2}$. The generator of the 1-parameter group $\mathbb{C} \ni t \mapsto M_{t a}^{\dagger}$ coincides with the operator of multiplication by the $L_{\chi}^{\infty}$-valued function

$$
\bar{\phi}_{\mathrm{a}}: \mathfrak{U}_{\pi} \ni u \mapsto \bar{\phi}_{\mathrm{a}}(u) \quad \text { where } \quad d M_{t \mathrm{a}}^{\dagger} /\left.d t\right|_{t=0}=\bar{\phi}_{\mathrm{a}} .
$$

The continuity of $E \ni a \mapsto \exp \left(\bar{\phi}_{\mathrm{a}}\right)$ implies that this 1-parameter group $M_{t a}^{\dagger}$ is strongly continuous on $H_{\chi}^{2}$. As a consequnce, its generator $\left(\bar{\phi}_{\mathrm{a}} f\right)(u)=$ $\bar{\phi}_{\mathrm{a}}(u) f(u)$ with domain $\mathfrak{D}\left(\bar{\phi}_{\mathrm{a}}\right)=\left\{f \in H_{\chi}^{2}: \bar{\phi}_{\mathrm{a}} f \in H_{\chi}^{2}\right\}$ is closed and denselydefined. As well, its power $\bar{\phi}_{\mathrm{a}}^{m}$ defined on $\mathfrak{D}\left(\bar{\phi}_{\mathrm{a}}^{m}\right)=\left\{f \in H_{\chi}^{2}: \bar{\phi}_{\mathrm{a}}^{m} f \in H_{\chi}^{2}\right\}$ for any $m \in \mathbb{N}$ is the same (see, e.g. [7] for details).

The additive group $(E,+)$ may be also linearly represented on $H_{\mathrm{w}}^{2}$ as the shift group

$$
T_{\mathrm{a}} \widehat{f}(x)=\widehat{f}(x+\mathrm{a}), \quad f \in H_{\chi}^{2}, \quad x, \mathrm{a} \in E .
$$

The directional derivative on the space $H_{\mathrm{w}}^{2}$ along a nonzero a $\in E$ coincides with the generator of the 1-parameter shift subgroup $\mathbb{C} \ni t \mapsto T_{t a}$, that is,

$$
\mathfrak{d}_{\mathrm{a}} \widehat{f}=\lim _{t \rightarrow 0} t^{-1}\left(T_{t \mathrm{a}} \widehat{f}-\widehat{f}\right) \quad \text { with domain } \mathfrak{D}\left(\mathfrak{d}_{\mathrm{a}}\right):=\left\{\widehat{f} \in H_{\mathrm{w}}^{2}: \mathfrak{d}_{\mathrm{a}} \widehat{f} \in H_{\mathrm{w}}^{2}\right\} .
$$

Note that the 1-parameter shift group $T_{t a}$, which is intertwined with $M_{t a}^{\dagger}$ by the $\mathcal{F}$-transform

$$
T_{t \mathrm{a}} \widehat{f}(x)=\int \exp \left[\bar{\phi}_{x+t \mathrm{a}}\right] f d \chi=\int \exp \left(\bar{\phi}_{x}\right) M_{t \mathrm{a}}^{\dagger} f d \chi
$$

is strongly continuous on $H_{\mathrm{w}}^{2}$. Since $\mathfrak{D}\left(\mathfrak{d}_{\mathrm{a}}^{m}\right)$ contains all polynomials from $H_{\mathrm{w}}^{2}$, each operator $\mathfrak{d}_{\mathrm{a}}^{m}$ with domain $\mathfrak{D}\left(\mathfrak{d}_{\mathrm{a}}^{m}\right)=\left\{\widehat{f} \in H_{\mathrm{w}}^{2}: \mathfrak{d}_{\mathrm{a}}^{m} \widehat{f} \in H_{\mathrm{w}}^{2}\right\}$ is closed and densely-defined. From (15) it directly follows

$$
\mathfrak{d}_{\mathrm{a}}^{m} \widehat{f}(x)=\left.\int \exp \left(\bar{\phi}_{x}\right) \frac{d^{m} M_{t \mathrm{a}}^{\dagger}}{d t^{m}}\right|_{t=0} f d \chi=\int \exp \left(\bar{\phi}_{x}\right) \bar{\phi}_{\mathrm{a}}^{m} f d \chi
$$

for all $f \in \mathfrak{D}\left(\bar{\phi}_{\mathrm{a}}^{m}\right)$ and $x \in E$. On the other hand, by Theorem 2 we have

$$
T_{\mathrm{a}} \widehat{f}(x)=\left\langle\mathcal{T}_{\mathrm{a}} \varepsilon(x) \mid \Phi^{*} f\right\rangle=\left\langle\varepsilon(x) \mid \mathcal{T}_{\mathrm{a}}^{*} \Phi^{*} f\right\rangle=\int \exp \left(\bar{\phi}_{x}\right) \Phi \mathcal{T}_{\mathrm{a}}^{*} \Phi^{*} f d \chi .
$$

Theorem 2 together with (15) and (17) imply that $M_{\mathrm{a}}^{\dagger}$ is connected with the exponential annihilation group $\mathcal{T}_{\mathrm{a}}^{*}$ by the intertwining operator $\Phi$. This can be written as $M_{\mathrm{a}}^{\dagger}=\Phi \mathcal{T}_{\mathrm{a}}^{*} \Phi^{*}$. Thus, the $\mathcal{F}$-transform serves as an intertwining 
operator for the groups $M_{\mathrm{a}}^{\dagger}$ on $H_{\chi}^{2}$. Moreover, using (15), (16) and (17), we obtain

$$
d^{m} T_{t \mathrm{a}} \widehat{f}(x) /\left.d t^{m}\right|_{t=0}=\left\langle\varepsilon(x) \mid \delta_{\mathrm{a}}^{* m} \Phi^{*} f\right\rangle_{\mathrm{w}}=\mathfrak{d}_{\mathrm{a}}^{m} \widehat{f}(x) .
$$

As a result, we have proved the following statement.

Theorem 3. For every $f \in H_{\chi}^{2}$ the following equalities hold,

$$
T_{\mathrm{a}} \mathcal{F}(f)=\mathcal{F}\left(M_{\mathrm{a}}^{\dagger} f\right), \quad M_{\mathrm{a}}^{\dagger} f=\Phi \mathcal{T}_{\mathrm{a}}^{*} \Phi^{*} f, \quad \mathrm{a} \in E,
$$

Moreover, for every $f \in \mathfrak{D}\left(\bar{\phi}_{\mathrm{a}}^{m}\right)(m \in \mathbb{N})$ and a nonzero $\mathrm{a} \in E$,

$$
\mathfrak{d}_{\mathrm{a}}^{m} \widehat{f}(x)=\left\langle\varepsilon(x) \mid \delta_{\mathrm{a}}^{* m} \Phi^{*} f\right\rangle_{\mathrm{w}}=\int \exp \left(\bar{\phi}_{x}\right) \bar{\phi}_{\mathrm{a}}^{m} f d \chi, \quad x \in E .
$$

Let us consider on $H_{\mathrm{w}}^{2}$ the multiplicative group with a nonzero a $\in E$,

$$
M_{\mathrm{a}^{*}} \widehat{f}(x)=\widehat{f}(x) \exp \langle x \mid \mathrm{a}\rangle, \quad \widehat{f} \in H_{\mathrm{w}}^{2} .
$$

The generator on $H_{\mathrm{w}}^{2}$ of the appropriate 1-parameter subgroup $\mathbb{C} \ni t \mapsto M_{\mathrm{ta}^{*}}$ is

$$
d M_{t \mathrm{a}^{*}} /\left.d t\right|_{t=0}=\langle\cdot \mid \mathrm{a}\rangle:=\mathrm{a}^{*}, \quad \mathrm{a} \in E .
$$

Hence, it coincides with the following linear operator of multiplication

$$
\left(\mathrm{a}^{*} \widehat{f}\right)(x)=\langle x \mid \mathrm{a}\rangle \widehat{f}(x) \quad \text { with domain } \quad \mathfrak{D}\left(\mathrm{a}^{*}\right)=\left\{\widehat{f} \in H_{\mathrm{w}}^{2}: \mathrm{a}^{*} \widehat{f} \in H_{\mathrm{w}}^{2}\right\} .
$$

Its power $\mathrm{a}^{* m}$ is densely-defined on $\mathfrak{D}\left(\mathrm{a}^{* m}\right)=\left\{\widehat{f} \in H_{\mathrm{w}}^{2}: \mathrm{a}^{* m} \widehat{f} \in H_{\mathrm{w}}^{2}\right\}$ which contains all polynomials from $H_{\mathrm{w}}^{2}$.

Using Lemma 3 we can represent the additive group $(E,+)$ over the space $H_{\chi}^{2}$ by the shift group

$$
T_{\mathrm{a}}^{\dagger}=\Phi \mathcal{T}_{\mathrm{a}} \Phi^{*} \quad \text { with the generator } \quad \delta_{\mathrm{a}}^{\dagger}=\Phi \delta_{\mathrm{a}} \Phi^{*}
$$

defined on $\mathfrak{D}\left(\delta_{\mathrm{a}}^{\dagger}\right)=\left\{f \in H_{\chi}^{2}: \delta_{\mathrm{a}}^{\dagger} f \in H_{\chi}^{2}\right\}$ This means that $T_{\mathrm{a}}^{\dagger}$ is connected via the intertwining operator $\Phi$ with the exponential creation group $\mathcal{T}_{\mathrm{a}}$.

Theorem 4. For every $f \in H_{\chi}^{2}$ the following equality holds,

$$
M_{\mathrm{a}} * \mathcal{F}(f)=\mathcal{F}\left(T_{\mathrm{a}}^{\dagger} f\right), \quad \mathrm{a} \in E,
$$

that is, the $\mathcal{F}$-transform is an intertwining operator for the groups $M_{\mathrm{a}^{*}}$ on $H_{\mathrm{w}}^{2}$ and $T_{\mathrm{a}}^{\dagger}$ on $H_{\chi}^{2}$. Moreover, for every $f \in \mathfrak{D}\left(\delta_{\mathrm{a}}^{\dagger m}\right)=\left\{f \in H_{\chi}^{2}: \delta_{\mathrm{a}}^{\dagger m} f \in H_{\chi}^{2}\right\}$ $(m \in \mathbb{N})$ and a nonzero $\mathrm{a} \in E$,

$$
\left(\mathrm{a}^{* m} \widehat{f}\right)(x)=\left\langle\varepsilon(x) \mid \delta_{\mathrm{a}}^{m} \Phi^{*} f\right\rangle_{\mathrm{w}}=\int \exp \left(\bar{\phi}_{x}\right) \delta_{\mathrm{a}}^{\dagger m} f d \chi, \quad x \in E .
$$

Proof. The equality (13) yields $\langle x \mid \mathrm{a}\rangle^{m} \psi_{n-m}^{*}(x)=\left\langle\delta_{\mathrm{a}, n}^{* m} x^{\otimes n} \mid \psi_{n-m}\right\rangle_{\mathrm{w}}$ for all $n \geq m$. By Theorem 2 for any $f=\sum_{n} f_{n} \in H_{\chi}^{2}$ there exists a unique 
$\psi=\bigoplus_{n} \psi_{n}$ in $\Gamma_{\mathrm{w}}$ with $\psi_{n} \in E^{\odot n}$ such that $\Phi^{*} f=\psi$ and $f_{n}=\psi_{n}^{*}$. Summing over all $m \in \mathbb{Z}_{+}$and $n \geq m$ and using (14), we obtain that

$$
\begin{aligned}
M_{\mathrm{a}^{*}} \widehat{f}(x) & =\exp \langle x \mid \mathrm{a}\rangle\left\langle\varepsilon(x) \mid \Phi^{*} f\right\rangle_{\mathbb{w}}=\sum_{m \in \mathbb{Z}_{+}} \frac{\langle x \mid \mathrm{a}\rangle^{m}}{m !} \sum_{n \geq m} \psi_{n-m}^{*}(x) \\
& =\left\langle\mathcal{T}_{\mathrm{a}}^{*} \varepsilon(x) \mid \Phi^{*} f\right\rangle_{\mathbb{w}}=\left\langle\varepsilon(x) \mid \mathcal{T}_{\mathrm{a}} \Phi^{*} f\right\rangle_{\mathbb{w}} .
\end{aligned}
$$

By Theorem 2 and Lemma 3 it follows that the equalities

$$
M_{t \mathrm{a}} \widehat{f}(x)=\left\langle\varepsilon(x) \mid \mathcal{T}_{t \mathrm{a}} \Phi^{*} f\right\rangle_{\mathrm{w}}=\int \exp \left(\bar{\phi}_{x}\right) T_{t \mathrm{a}}^{\dagger} f d \chi, \quad t \in \mathbb{C}
$$

hold for all $\widehat{f} \in H_{\mathrm{w}}^{2}$. On the other hand, the equalities (14) and (19) yield

$$
\left.\frac{d^{m} M_{t \mathrm{a}^{*}} \widehat{f}(x)}{d t^{m}}\right|_{t=0}=\left.\int \exp \left(\bar{\phi}_{x}\right) \frac{d^{m} T_{t \mathrm{a}}^{\dagger}}{d t^{m}}\right|_{t=0} f d \chi=\int \exp \left(\bar{\phi}_{x}\right) \delta_{\mathrm{a}}^{\dagger m} f d \chi
$$

for all $f \in \mathfrak{D}\left(\delta_{\mathrm{a}}^{\dagger m}\right)$. This in turn yields (18).

\section{Commutation Relations}

Describe the commutation relations between $M_{\mathrm{a}}^{\dagger}$ and $T_{\mathrm{b}}^{\dagger}$ on the Hardy space $H_{\chi}^{2}$.

Theorem 5. For any nonzero $\mathrm{a}, \mathrm{b} \in E$ the commutation relations

$$
M_{\mathrm{a}}^{\dagger} T_{\mathrm{b}}^{\dagger}=\exp \langle\mathrm{a} \mid \mathrm{b}\rangle T_{\mathrm{b}}^{\dagger} M_{\mathrm{a}}^{\dagger}, \quad\left(\bar{\phi}_{\mathrm{a}} \delta_{\mathrm{b}}^{\dagger}-\delta_{\mathrm{b}}^{\dagger} \bar{\phi}_{\mathrm{a}}\right) f=\langle\mathrm{a} \mid \mathrm{b}\rangle f
$$

hold, wherein $f$ belongs to the dense subspace $\mathfrak{D}\left(\bar{\phi}_{\mathrm{b}}^{2}\right) \cap \mathfrak{D}\left(\delta_{\mathrm{a}}^{\dagger 2}\right) \subset H_{\chi}^{2}$.

Proof. Let us prove that the following equalities hold,

$$
T_{\mathrm{a}} M_{\mathrm{b}^{*}}=\exp \langle\mathrm{a} \mid \mathrm{b}\rangle M_{\mathrm{b}^{*}} T_{\mathrm{a}}, \quad\left(\mathfrak{d}_{\mathrm{a}} \mathrm{b}^{*}-\mathrm{b}^{*} \mathfrak{d}_{\mathrm{a}}\right) \widehat{f}=\langle\mathrm{a} \mid \mathrm{b}\rangle \widehat{f}
$$

where $\widehat{f} \in \mathfrak{D}\left(\mathrm{b}^{* 2}\right) \cap \mathfrak{D}\left(\mathfrak{d}_{\mathrm{a}}^{2}\right)$. First property follows from the direct calculations:

$$
\begin{aligned}
& M_{\mathrm{b}^{*}} T_{\mathrm{a}} \widehat{f}(x)=\exp \langle x \mid \mathrm{b}\rangle \widehat{f}(x+\mathrm{a}), \\
& T_{\mathrm{a}} M_{\mathrm{b}^{*}} \widehat{f}(x)=\widehat{f}(x+\mathrm{a}) \exp \langle x \mid \mathrm{b}\rangle \exp \langle\mathrm{a} \mid \mathrm{b}\rangle=\exp \langle\mathrm{a} \mid \mathrm{b}\rangle M_{\mathrm{b}^{*}} T_{\mathrm{a}} \widehat{f}(x)
\end{aligned}
$$

for all $\widehat{f} \in H_{\mathrm{w}}^{2}$ and $x \in E$. For any $\widehat{f} \in \mathfrak{D}\left(\mathrm{b}^{* 2}\right) \cap \mathfrak{D}\left(\mathfrak{d}_{\mathrm{a}}^{2}\right)$ and $t \in \mathbb{C}$, we have

$$
\begin{aligned}
\left.\frac{d^{2}}{d t^{2}} T_{t \mathrm{a}} M_{t \mathrm{~b}} * \widehat{f}\right|_{t=0} & =\left[\mathfrak{d}_{\mathrm{a}}^{2} T_{t \mathrm{a}} M_{t \mathrm{~b}} * \widehat{f}+2 \mathfrak{d}_{\mathrm{a}} T_{t \mathrm{a}} \mathrm{b}^{*} M_{t \mathrm{~b}} * \widehat{f}+T_{t \mathrm{a}} \mathrm{b}^{* 2} M_{t \mathrm{~b}} * \widehat{f}\right]_{t=0} \\
& =\left(\mathfrak{d}_{\mathrm{a}}^{2}+2 \mathfrak{d}_{\mathrm{a}} \mathrm{b}^{*}+\mathrm{b}^{* 2}\right) \widehat{f}
\end{aligned}
$$


On the other hand, differentiating again, we have

$$
\begin{aligned}
\left.\frac{d}{d t} T_{t \mathrm{a}} M_{t \mathrm{~b}^{*}} \widehat{f}\right|_{t=0}= & {\left[\frac{d}{d t} \exp \langle t \mathrm{a} \mid \bar{t} \mathrm{~b}\rangle M_{t \mathrm{~b} *} T_{t \mathrm{a}} \widehat{f}+\exp \langle t \mathrm{a} \mid \bar{t} \mathrm{~b}\rangle \frac{d}{d t} M_{t \mathrm{~b}} * T_{t \mathrm{a}} \widehat{f}\right]_{t=0}, } \\
\left(\mathfrak{d}_{\mathrm{a}}^{2}+2 \mathfrak{d}_{\mathrm{a}} \mathrm{b}^{*}+\mathrm{b}^{* 2}\right) \widehat{f}= & \frac{d}{d t}\left[\frac{d}{d t} T_{t \mathrm{a}} M_{t \mathrm{~b}} * \widehat{f}\right]_{t=0}=\left[\frac{d^{2}}{d t^{2}} \exp \langle t \mathrm{a} \mid \bar{t} \mathrm{~b}\rangle M_{t \mathrm{~b}} * T_{t \mathrm{a}} \widehat{f}\right. \\
& +2 \frac{d}{d t} \exp \langle t \mathrm{a} \mid \bar{t} \mathrm{~b}\rangle \frac{d}{d t} M_{t \mathrm{~b} *} T_{t \mathrm{a}} \widehat{f} \\
& \left.+\exp \langle t \mathrm{a} \mid \bar{t} \mathrm{~b}\rangle \frac{d^{2}}{d t^{2}} M_{t \mathrm{~b} *} T_{t \mathrm{a}} \widehat{f}\right]_{t=0} \\
= & 2\langle\mathrm{a} \mid \mathrm{b}\rangle \widehat{f}+\left(\mathfrak{d}_{\mathrm{a}}^{2}+2 \mathrm{~b}^{*} \mathfrak{d}_{\mathrm{a}}+\mathrm{b}^{* 2}\right) \widehat{f} .
\end{aligned}
$$

This yields $(20)$ where $\mathfrak{D}\left(\mathrm{b}^{* 2}\right) \cap \mathfrak{D}\left(\mathfrak{d}_{\mathrm{a}}^{2}\right)$ contains the dense subspace in $H_{\mathrm{w}}^{2}$ of all polynomials $\widehat{f}$ generating by finite sums $\Phi^{*}(f)=\bigoplus_{n} \psi_{n} \in \Gamma_{\mathrm{w}}$.

From $M_{\mathrm{b} *} \widehat{f}(x)=\left\langle\varepsilon(x) \mid \mathcal{T}_{\mathrm{b}} \Phi^{*} f\right\rangle_{\mathrm{w}}$ it follows that $\widehat{I} \mathcal{T}_{\mathrm{b}}=M_{\mathrm{a}^{*}} \widehat{I}$ with $\widehat{I}:=$ $\mathcal{F} \Phi$. Thus, $T_{\mathrm{b}}^{\dagger}=\Phi \mathcal{T}_{\mathrm{b}} \Phi^{*}=\Phi \widehat{I}^{-1} M_{\mathrm{b}^{*}} \widehat{I} \Phi^{*}=\mathcal{F}^{-1} M_{\mathrm{b}^{*}} \mathcal{F}$. Using that $M_{\mathrm{a}}^{\dagger}=$ $\mathcal{F}^{-1} T_{\mathrm{a}} \mathcal{F}$ with $\mathcal{F}^{-1}: H_{\mathrm{w}}^{2} \rightarrow H_{\chi}^{2}$ and applying (20), we obtain

$$
\begin{aligned}
& M_{\mathrm{a}}^{\dagger} T_{\mathrm{b}}^{\dagger}=\mathcal{F}^{-1} T_{\mathrm{a}} M_{\mathrm{b}^{*}} \mathcal{F}=\exp \langle x \mid \mathrm{b}\rangle \mathcal{F}^{-1} M_{\mathrm{b}^{*}} T_{\mathrm{a}} \mathcal{F}=\exp \langle x \mid \mathrm{b}\rangle T_{\mathrm{b}}^{\dagger} M_{\mathrm{a}}^{\dagger}, \\
& \left(\bar{\phi}_{\mathrm{a}} \delta_{\mathrm{b}}^{\dagger}-\delta_{\mathrm{b}}^{\dagger} \bar{\phi}_{\mathrm{a}}\right) f=\mathcal{F}^{-1}\left(\mathfrak{d}_{\mathrm{a}} \mathrm{b}^{*}-\mathrm{b}^{*} \mathfrak{d}_{\mathrm{a}}\right) \mathcal{F} f=\langle\mathrm{a} \mid \mathrm{b}\rangle f
\end{aligned}
$$

for all $f \in \mathfrak{D}\left(\bar{\phi}_{\mathrm{b}}^{2}\right) \cap \mathfrak{D}\left(\delta_{\mathrm{a}}^{\dagger 2}\right)$. For any $f=\sum_{n} f_{n} \in H_{\chi}^{2}$ there exists a unique $\psi=\bigoplus_{n} \psi_{n}$ in $\Gamma_{\mathrm{w}}$ with $\psi_{n} \in E_{\mathrm{w}}^{\odot n}$ such that the equalities $\Phi^{*} f=\psi$ and $f_{n}=$ $\psi_{n}^{*}$ hold. Hence, the following embedding $\mathfrak{D}\left(\bar{\phi}_{\mathrm{b}}^{2}\right) \cap \mathfrak{D}\left(\delta_{\mathrm{a}}^{\dagger 2}\right) \subset H_{\chi}^{2}$ is dense.

\section{Gauss-Weierstrass Semigroups}

Next we show that the 1-parameter Gauss-Weierstrass semigroups on the Hardy space $H_{\chi}^{2}$ can be well described by shift and multiplicative groups (a classic case can be found in $[7$, n.4.3.2]). For this purpose we use the Gaussian kernel

$$
\mathfrak{g}_{r}(\tau)=\frac{1}{\sqrt{4 \pi r}} \exp \left(-\frac{\tau^{2}}{4 r}\right), \quad \tau \in \mathbb{R}, \quad r>0
$$

Theorem 6. The 1-parameter Gauss-Weierstrass semigroups $\left\{W_{r}^{\delta_{a}^{\dagger}}: r>0\right\}$ and $\left\{W_{r}^{\bar{\phi}_{\mathrm{a}}}: r>0\right\}$, defined on the Hardy space $H_{\chi}^{2}$ for any nonzero a $\in E$ as

$$
W_{r}^{\delta_{\mathrm{a}}^{\dagger}} f=\int_{\mathbb{R}} \mathfrak{g}_{r}(\tau) T_{\tau \mathrm{a}}^{\dagger} f d \tau \quad \text { and } \quad W_{r}^{\overline{\mathrm{a}}_{\mathrm{a}}} f=\int_{\mathbb{R}} \mathfrak{g}_{r}(\tau) M_{\tau \mathrm{a}}^{\dagger} f d \tau, \quad f \in H_{\chi}^{2},
$$

are generated by $\delta_{\mathrm{a}}^{\dagger 2}$ and $\bar{\phi}_{\mathrm{a}}^{2}$, respectively. 
Proof. First it is sufficient to prove that the axillary 1-parameter families of linear operators over $H_{\mathrm{w}}^{2}$

$$
G_{r}^{\mathrm{a}^{*}} \widehat{f}=\int_{\mathbb{R}} \mathfrak{g}_{r}(\tau) M_{\tau \mathrm{a}^{*}} \widehat{f} d \tau \quad \text { and } \quad G_{r}^{\partial_{\mathrm{a}}} \widehat{f}=\int_{\mathbb{R}} \mathfrak{g}_{r}(\tau) T_{\tau \mathrm{a}} \widehat{f} d \tau, \quad \widehat{f} \in H_{\mathrm{w}}^{2}
$$

can be generated by $\mathrm{a}^{* 2}$ and $\mathfrak{d}_{\mathrm{a}}^{2}$ and satisfy the semigroup property. Properties of Gaussian kernel yield

$$
\begin{aligned}
\int_{\mathbb{R}} \mathfrak{g}_{r}(\tau) \tau^{2 k} d \tau & =\left.\frac{1}{2 \sqrt{\pi r}} \int_{\mathbb{R}} e^{-\frac{\tau^{2}}{4 r}} \tau^{2 k} d \tau\right|_{\tau=2 \sqrt{r} v}=\frac{(2 \sqrt{r})^{2 k}}{\sqrt{\pi}} \int_{\mathbb{R}} e^{-v^{2} v^{2 k} d v} \\
& =\frac{2^{2 k} r^{k}}{\sqrt{\pi}} \Gamma\left(\frac{2 k+1}{2}\right)=\frac{2(2 k-1) !}{(k-1) !} r^{k}, \quad k \in \mathbb{N} .
\end{aligned}
$$

We can rewrite $G_{r}^{a^{*}} \widehat{f}$ on the dense subspace $\left\{\widehat{f} \in H_{\mathrm{w}}^{2}: \exp \left(\tau \mathrm{a}^{*}\right) \widehat{f} \in H_{\mathrm{w}}^{2}\right\}$ as

$$
\begin{aligned}
G_{r}^{a^{*}} \widehat{f} & =\int_{\mathbb{R}} \mathfrak{g}_{r}(\tau) \exp \left(\tau \mathrm{a}^{*}\right) \widehat{f} d \tau=\sum_{l \in \mathbb{Z}_{+}} \frac{\mathrm{a}^{* l} \widehat{f}}{l !} \int_{\mathbb{R}} \mathfrak{g}_{r}(\tau) \tau^{l} d \tau \\
& =\sum_{k \in \mathbb{Z}_{+}} \frac{2(2 k-1) !}{(k-1) !} \frac{r^{k} \mathrm{a}^{* 2 k} \widehat{f}}{(2 k) !}=\sum_{k \in \mathbb{Z}_{+}} \frac{r^{k} \mathrm{a}^{* 2 k} \widehat{f}}{k !}=\exp \left(r \mathrm{a}^{* 2}\right) \widehat{f}
\end{aligned}
$$

By first equality in (22) the family $G_{r}^{\mathrm{a}^{*}}$ can be extended to the convolution

$$
\mathfrak{g}_{r} \circledast \widehat{f}:=\int_{\mathbb{R}} \mathfrak{g}_{r}(\tau) M_{\tau \mathbf{a}^{*}} \widehat{f} d \tau, \quad \widehat{f} \in H_{\mathrm{w}}^{2}
$$

(dependent on a) over the whole space $H_{\mathrm{w}}^{2}$. Thus, to show that the semigroup property holds, it suffices to show that

$$
\mathfrak{g}_{r+s} \circledast \widehat{f}=G_{r+s}^{\mathrm{a}^{*}} \widehat{f}=\left(G_{r}^{\mathrm{a}^{*}} \circ G_{s}^{\mathrm{a}^{*}}\right) \widehat{f}=\mathfrak{g}_{r} \circledast\left(\mathfrak{g}_{s} \circledast \widehat{f}\right)=\left(\mathfrak{g}_{r} * \mathfrak{g}_{s}\right) \circledast \widehat{f} .
$$

But this straightly follows from the known convolution equality $\mathfrak{g}_{r+s}=\mathfrak{g}_{r} * \mathfrak{g}_{s}$.

Further, using the equality $T_{\mathrm{a}}^{\dagger}=\mathcal{F}^{-1} M_{\mathrm{a}} \mathcal{F}$ we obtain that

$$
W_{r}^{\delta_{\mathrm{a}}^{\dagger}} f=\int_{\mathbb{R}} \mathfrak{g}_{r}(\tau) \mathcal{F}^{-1} M_{\tau \mathrm{a}^{*}} \mathcal{F} f d \tau=\mathcal{F}^{-1} G_{r}^{\mathrm{a}^{*}} \mathcal{F} f
$$

for all $f \in H_{\chi}^{2}$. By Theorem 4 it follows that

$$
\left.\frac{d W_{r}^{\delta_{\mathrm{a}}^{\dagger}} f}{d r}\right|_{r=0}=\left.\mathcal{F}^{-1} \frac{G_{r}^{\mathrm{a}^{*} \widehat{f}}}{d r}\right|_{r=0}=\mathcal{F}^{-1} \mathrm{a}^{* 2} \widehat{f}=\delta_{\mathrm{a}}^{\dagger 2} f
$$

for all $f \in \mathfrak{D}\left(\delta_{\mathrm{a}}^{\dagger 2}\right)$, since $\widehat{f} \in \mathfrak{D}\left(\mathrm{a}^{* 2}\right)$ and $\delta_{\mathrm{a}}^{\dagger 2}=\mathcal{F}^{-1} \mathrm{a}^{* 2} \mathcal{F}$. Hence, the case of semigroup $W_{r}^{\delta_{a}^{\dagger}}$ is proven.

Similar reasonings can be applied to the semigroup $G_{r}^{\partial_{a}}$. As a result, we obtain that the equalities $W_{r}^{\bar{\phi}_{\mathrm{a}}}=\mathcal{F}^{-1} G_{r}^{\partial_{\mathrm{a}}} \mathcal{F}$ and $\bar{\phi}_{\mathrm{a}}^{2}=\mathcal{F}^{-1} \mathfrak{d}_{\mathrm{a}}^{2} \mathcal{F}$ hold. 


\section{Complexified Infinite-Dimensional Heisenberg Group}

Let us give yet another application. Consider an infinite-dimensional analog of the Heisenberg group over $\mathbb{C}$. Namely, let us define the group $\mathfrak{G}$ of upper triangular matrix-type elements

$$
X(\mathrm{a}, \mathrm{b}, t)=\left[\begin{array}{lll}
1 & \mathrm{a} & t \\
0 & 1 & \mathrm{~b} \\
0 & 0 & 1
\end{array}\right], \quad t \in \mathbb{C}, \quad \mathrm{a}, \mathrm{b} \in E
$$

with unit $X(0,0,0)$ and multiplication

$$
\left[\begin{array}{ccc}
1 & \mathrm{a} & t \\
0 & 1 & \mathrm{~b} \\
0 & 0 & 1
\end{array}\right]\left[\begin{array}{ccc}
1 & \mathrm{a}^{\prime} & t^{\prime} \\
0 & 1 & \mathrm{~b}^{\prime} \\
0 & 0 & 1
\end{array}\right]=\left[\begin{array}{ccc}
1 & \mathrm{a}+\mathrm{a}^{\prime} & t+t^{\prime}+\left\langle\mathrm{a} \mid \mathrm{b}^{\prime}\right\rangle \\
0 & 1 & \mathrm{~b}+\mathrm{b}^{\prime} \\
0 & 0 & 1
\end{array}\right] .
$$

Obviously, $X(\mathrm{a}, \mathrm{b}, t)^{-1}=X(-\mathrm{a},-\mathrm{b},-t+\langle\mathrm{a} \mid \mathrm{b}\rangle)$.

We will now describe an irreducible linear representation of the group $\mathfrak{G}$. For this purpose we will use the algebra $\mathbb{H}$ of quaternions $\gamma=\alpha_{1}+\alpha_{2} \dot{\mathbb{1}}+\beta_{1} \dot{\mathfrak{j}}$ $+\beta_{2} \mathbb{k}=\left(\alpha_{1}+\alpha_{2} \dot{i}\right)+\left(\beta_{1}+\beta_{2} \dot{i}\right) \dot{j}=\alpha+\beta \dot{j}$ as pairs of complex numbers $(\alpha, \beta) \in \mathbb{C}^{2}$ with $\alpha=\alpha_{1}+\alpha_{2} \dot{\mathrm{i}}, \beta=\beta_{1}+\beta_{2} \dot{\mathrm{i}} \in \mathbb{C}$ and $\alpha_{\imath}, \beta_{\imath} \in \mathbb{R}(\iota=1,2)$ where basis elements in $\mathbb{R}^{4}$ satisfy the relations $\dot{\mathrm{i}}^{2}=\dot{\mathrm{j}}^{2}=\mathbb{k}^{2}=\mathbf{i} \mathbf{j} \mathbb{k}=-1$,

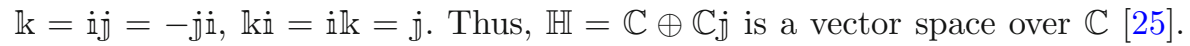
Denote $\beta:=\Im \gamma$ where $\gamma=\alpha+\beta \dot{j}$.

Let $E_{\mathbb{H}}=E \oplus E_{\mathfrak{j}}$ be the Hilbert space with $\mathbb{H}$-valued scalar product

$$
\left\langle p \mid p^{\prime}\right\rangle=\left\langle a+b j \mid a^{\prime}+b^{\prime} j\right\rangle=\left\langle a \mid a^{\prime}\right\rangle+\left\langle b \mid b^{\prime}\right\rangle+\left[\left\langle a^{\prime} \mid b\right\rangle-\left\langle a \mid b^{\prime}\right\rangle\right] j
$$

where $p=a+b j$ with $a, b \in E$ (similarly, for $\left.p^{\prime}=a^{\prime}+b^{\prime} j\right)$. Hence,

$$
\Im\left\langle p \mid p^{\prime}\right\rangle=\left\langle a^{\prime} \mid b\right\rangle-\left\langle a \mid b^{\prime}\right\rangle, \quad \Im\langle p \mid p\rangle=0 .
$$

The following theorem describes a representation of the above infinitedimensional Heisenberg group which can be seen as an analog of the WeylSchrödinger representation

Theorem 7. The linear representation of $\mathfrak{G}$ over $H_{\chi}^{2}$

$$
\mathscr{W}^{\dagger}: \mathfrak{G} \ni X(\mathrm{a}, \mathrm{b}, t) \longmapsto \exp \left[t+\frac{1}{2}\langle\mathrm{a} \mid \mathrm{b}\rangle\right] T_{\mathrm{a}}^{\dagger} M_{\mathrm{b}}^{\dagger}
$$

is well defined and irreducible.

Proof. First we prove that the following operator representation

$$
\mathscr{W}: \mathfrak{G} \ni X(\mathrm{a}, \mathrm{b}, t) \longmapsto \exp \left[t+\frac{1}{2}\langle\mathrm{a} \mid \mathrm{b}\rangle\right] M_{\mathrm{a}^{*}} T_{\mathrm{b}}
$$

into the algebra of all bounded linear operator on $H_{\mathrm{w}}^{2}$ is well defined and irreducible. Consider the auxiliary group $\mathbb{C} \times E_{\mathbb{H}}$ with the multiplication

$$
(t, \mathrm{p})\left(t^{\prime}, \mathrm{p}^{\prime}\right)=\left(t+t^{\prime}-\frac{1}{2} \Im\left\langle\mathrm{p} \mid \mathrm{p}^{\prime}\right\rangle, \mathrm{p}+\mathrm{p}^{\prime}\right)
$$


for all $\mathrm{p}=\mathrm{a}+\mathrm{b} \dot{\mathfrak{j}}, \mathrm{p}^{\prime}=\mathrm{a}^{\prime}+\mathrm{b}^{\prime} \mathfrak{j} \in E_{\mathbb{H}}$. It is related to $\mathfrak{G}$ via the mapping

$$
\mathscr{G}: X(\mathrm{a}, \mathrm{b}, t) \longmapsto\left(t-\frac{1}{2}\langle\mathrm{a} \mid \mathrm{b}\rangle, \mathrm{a}+\mathrm{bj}\right) .
$$

Check that $\mathscr{G}$ is a group isomorphism. In fact,

$$
\begin{aligned}
\mathscr{G} & \left(X(\mathrm{a}, \mathrm{b}, t) X\left(\mathrm{a}^{\prime}, \mathrm{b}^{\prime}, t^{\prime}\right)\right)=\mathscr{G}\left(X\left(\mathrm{a}+\mathrm{a}^{\prime}, \mathrm{b}+\mathrm{b}^{\prime}, t+t^{\prime}+\left\langle\mathrm{a} \mid \mathrm{b}^{\prime}\right\rangle\right)\right) \\
& =\left(t+t^{\prime}+\left\langle\mathrm{a} \mid \mathrm{b}^{\prime}\right\rangle-\frac{1}{2}\left[\left\langle\mathrm{a}+\mathrm{a}^{\prime} \mid \mathrm{b}+\mathrm{b}^{\prime}\right\rangle\right],\left(\mathrm{a}+\mathrm{a}^{\prime}\right)+\left(\mathrm{b}+\mathrm{b}^{\prime}\right) \dot{j}\right) \\
& =\left(t+t^{\prime}-\frac{1}{2}\left[\langle\mathrm{a} \mid \mathrm{b}\rangle+\left\langle\mathrm{a}^{\prime} \mid \mathrm{b}^{\prime}\right\rangle\right]+\frac{1}{2}\left[\left\langle\mathrm{a} \mid \mathrm{b}^{\prime}\right\rangle-\left\langle\mathrm{a}^{\prime} \mid \mathrm{b}\right\rangle\right],(\mathrm{a}+\mathrm{a})+\left(\mathrm{b}+\mathrm{b}^{\prime}\right) \dot{j}\right) \\
& =\left(t-\frac{1}{2}\langle\mathrm{a} \mid \mathrm{b}\rangle, \mathrm{a}+\mathrm{b} \dot{j}\right)\left(t^{\prime}-\frac{1}{2}\left\langle\mathrm{a}^{\prime} \mid \mathrm{b}^{\prime}\right\rangle, \mathrm{a}^{\prime}+\mathrm{b}^{\prime} \dot{\mathfrak{j}}\right) \\
& =\mathscr{G}(X(\mathrm{a}, \mathrm{b}, t)) \mathscr{G}\left(X\left(\mathrm{a}^{\prime}, \mathrm{b}^{\prime}, t^{\prime}\right)\right) .
\end{aligned}
$$

Now let us check that the Weyl-like operator

$$
W(\mathrm{p})=\exp \left[\frac{1}{2}\langle\mathrm{a} \mid \mathrm{b}\rangle\right] M_{\mathrm{a}} * T_{\mathrm{b}}, \quad \mathrm{p}=\mathrm{a}+\mathrm{b} \dot{j}
$$

on the space $H_{\mathrm{w}}^{2}$ satisfies the commutation relation

$$
W\left(\mathrm{p}+\mathrm{p}^{\prime}\right)=\exp \left[-\frac{1}{2} \Im\left\langle\mathrm{p} \mid \mathrm{p}^{\prime}\right\rangle\right] W(\mathrm{p}) W\left(\mathrm{p}^{\prime}\right) .
$$

In fact, using (20), we obtain

$$
\begin{aligned}
\exp & {\left[\frac{1}{2}\left\langle\mathrm{a} \mid \mathrm{b}^{\prime}\right\rangle-\frac{1}{2}\left\langle\mathrm{a}^{\prime} \mid \mathrm{b}\right\rangle\right] W(\mathrm{p}) W\left(\mathrm{p}^{\prime}\right) } \\
& =\exp \left[\frac{1}{2}\langle\mathrm{a} \mid \mathrm{b}\rangle+\frac{1}{2}\left\langle\mathrm{a}^{\prime} \mid \mathrm{b}^{\prime}\right\rangle\right] \exp \left[\frac{1}{2}\left\langle\mathrm{a} \mid \mathrm{b}^{\prime}\right\rangle-\frac{1}{2}\left\langle\mathrm{a}^{\prime} \mid \mathrm{b}\right\rangle\right] M_{\mathrm{a}^{*}} T_{\mathrm{b}} M_{\mathrm{a}^{\prime *}} T_{\mathrm{b}^{\prime}} \\
& =\exp \left[\frac{1}{2}\left\langle\mathrm{a}+\mathrm{a}^{\prime} \mid \mathrm{b}+\mathrm{b}^{\prime}\right\rangle\right] M_{\mathrm{a}^{*}+\mathrm{a}^{\prime *}} T_{\mathrm{b}+\mathrm{b}^{\prime}}=W\left(\mathrm{p}+\mathrm{p}^{\prime}\right) .
\end{aligned}
$$

As a consequence, the mapping $\mathscr{I}: \mathbb{C} \times E_{\mathbb{H}} \ni(t, \mathrm{p}) \longmapsto \exp (t) W(\mathrm{p})$ is a group isomorphism. So, $\mathscr{W}$ is also a group isomorphism as a composition of the group isomorphisms $\mathscr{I}$ and $\mathscr{G}$.

Let us check irreducibility. If there exists an element $x_{0} \neq 0$ in $E$ and an integer $n>0$ such that

$$
\exp \left[t+\frac{1}{2}\langle\mathrm{a} \mid \mathrm{b}\rangle\right] e^{\langle\mathrm{a} \mid x\rangle}\left[x_{0}^{*}(x+\mathrm{b})\right]^{n}=0 \text { for all } \quad x, \mathrm{a}, \mathrm{b} \in E
$$

then $x_{0}=0$. This gives a contradiction. Hence the representation $\mathscr{W}$ is irreducible. Finally, using that

$$
\exp \left[t+\frac{1}{2}\langle\mathrm{a} \mid \mathrm{b}\rangle\right] T_{\mathrm{a}}^{\dagger} M_{\mathrm{b}}^{\dagger}=\mathcal{F}^{-1}\left(\exp \left[t+\frac{1}{2}\langle\mathrm{a} \mid \mathrm{b}\rangle\right] M_{\mathrm{a} *} T_{\mathrm{b}}\right) \mathcal{F}
$$

we conclude that the group representation $\mathscr{W}^{\dagger}=\mathcal{F}^{-1} \mathscr{W} \mathcal{F}$ is irreducible. 


\section{Acknowledgements}

I am grateful to Referee for valuable suggestions which improved this article.

Open Access. This article is distributed under the terms of the Creative Commons Attribution 4.0 International License (http://creativecommons.org/licenses/ by/4.0/), which permits unrestricted use, distribution, and reproduction in any medium, provided you give appropriate credit to the original author(s) and the source, provide a link to the Creative Commons license, and indicate if changes were made.

\section{References}

[1] Beltiţă, I., Beltiţă, D.: On Weyl calculus in infinitely many variables. Geom. Methods Phys. In: Kielanowski, P., Buchstaber, V., Odzijewicz, A., Schlichenmaier, M., Voronov, T. (eds.) XXIX Workshop 2010, pp. 95-104 (2010)

[2] Beltiţă, I., Beltiţă, D.: Representation of nilpotent Lie groups via measurable dynamical systems. Geom. Methods Phys. In: XXXIV Workshop 2015 Trends in Mathematics, pp. 95-104 (2015)

[3] Beltiţă, I., Beltiţă, D., Măntoiu, M.: On Wigner transforms in infinite dimensions. J. Math. Phys. 57(021705), 1-13 (2016)

[4] Berezanski, Y.M., Kondratiev, Y.G.: Spectral Methods in Infinite-Dimensional Analysis. Springer, Berlin (1995)

[5] Borodin, A., Olshanski, G.: Harmonic analysis on the infinite-dimensional unitary group and determinantal point processes. Ann. Math. 161(3), 1319-1422 (2005)

[6] Bourbaki, N.: Integration II. Springer, Berlin (2004)

[7] Butzer, P.L., Berens, H.: Semi-groups of Operators and Approximation. Springer, Berlin (1967)

[8] Carando, D., Dimand, V., Muro, S.: Coherent sequences of polynomial ideals on Banach spaces. Math. Nachr. 282(8), 1111-1133 (2009)

[9] Cole, B., Gamelin, T.W.: Representing measures and Hardy spaces for the infinite polydisk algebra. Proc. Lond. Math. Soc. 53, 112-142 (1986)

[10] Dwyer, A.W.: Partial differential equationa in Fischer-Fock spaces for the Hilbert-Schmidt holomorphy type. Bull. Am. Soc. 77, 725-730 (1971)

[11] Floret, K.: Natural norms on symmetric tensor products of normed spaces. Note Mat. 17, 153-188 (1997)

[12] Holmes, I., Sengupta, A.N.: The Gaussian Radon transform in the classical Wiener space. Commun. Stoch. Anal. 8(2), 247-268 (2014)

[13] Klenke, A.: Probability Theory. A Comprehensive Course. Springer, Berlin (2008)

[14] Lopushansky, O., Zagorodnyuk, A.: Hilbert spaces of analytic functions of infinitely many variables. Ann. Pol. Math. 81(2), 111-122 (2003)

[15] Lopushansky, O.: The Hilbert-Schmidt analyticity associated with infinitedimensional unitary groups. Results Math. 71(1), 111-126 (2017) 
[16] Neeb, K.-H.: Holomorphy and Convexity in Lie Theory, vol. 28. De Gruyter Expositions in Mathematics, Berlin (2000)

[17] Neeb, K.-H., Ørted, B.: Hardy spaces in an infinite dimensional sett. In: Doebner, H.D. (ed.) Lie Theory and Its Applications in Physics, pp. 3-27. World Scientific Publishing, Singapore (1998)

[18] Neretin, Y.A.: Hua-type integrals over unitary groups and over projective limits of unitary groups. Duke Math. J. 114(2), 239-266 (2002)

[19] Okada, S., Okazaki, Y.: Projective limit of infinite radon measures. J. Aust. Math. Soc. 25(A), 328-331 (1978)

[20] Olshanski, G.: The problem of harmonic analysis on the infinite-dimensional unitary group. J. Funct. Anal. 205(2), 464-524 (2003)

[21] Petersson, P.: Hypercyclic convolution operators on entire functions of HilbertSchmidt holomorphy type. Ann. Math. Blaise Pascal 8(2), 107-114 (2001)

[22] Pinasco, D., Zalduendo, I.: Integral representations of holomorphic functions on Banach spaces. J. Math. Anal. Appl. 308, 159-174 (2005)

[23] Stroock, D.W.: Abstract Wiener space, revisited. Commun. Stoch. Anal. 2(1), 145-151 (2008)

[24] Tomas, E.: On Prohorov's criterion for projective limits. Oper. Theory Adv. Appl. 168, 251-261 (2006)

[25] Ward, J.: Quaternions and Cayley Numbers: Algebra and Applications. Kluwer Academic Publishers, Dordrecht (1997)

[26] Yamasaki, Y.: Projective limit of Haar measures on $\mathrm{O}(\mathrm{n})$. Publ. Res. Inst. Math. Sci. 8, 141-149 (1972)

Oleh Lopushansky

Faculty of Mathematics and Natural Sciences

University of Rzeszów

1 Pigonia Str.

35-310 Rzeszow

Poland

e-mail: ovlopusz@ur.edu.pl;

ovlopushansky@hotmail.com

Received: April 2, 2017.

Accepted: September 7, 2017. 